\title{
Theory and application of reverberated direct and indirect noise
}

\author{
E. O. Rolland ${ }^{1} \dagger$, F. De Domenico ${ }^{1}$ and S. Hochgreb ${ }^{1}$ \\ ${ }^{1}$ Department of Engineering, University of Cambridge, Trumpington Street, Cambridge CB2 1PZ, UK
}

(Received 5 September 2016; revised 15 March 2017; accepted 17 March 2017; first published online 27 April 2017)

The generation of a temperature disturbance in a flow is accompanied by the production of acoustic waves (direct noise) and of an entropy perturbation. If this entropy perturbation is accelerated or decelerated (as is the case through a nozzle or flow restriction), additional acoustic waves are generated (indirect noise). Several studies have demonstrated this mechanism in controlled conditions by using entropy wave generators, in which entropy waves are generated and convected through a nozzle, leading to direct and indirect noise. An analytical analysis of the direct and indirect noise produced by the generation and acceleration of entropy waves in a reflective environment is presented. The effect of reverberation (repeated acoustic reflections) on low-frequency perturbations (characteristic of entropy wave generators) is determined analytically. These results are then implemented in a set of limit cases, showing the limit behaviours of such systems. The analytical model is applied to the case of the Cambridge entropy wave generator experiment, in which entropy waves are generated by an electric heater and accelerated through a subsonic orifice plate. Due to the clear time separation of direct and indirect noise in the experimental results, direct and indirect noise transfer functions can be extracted from the experimental data for the first time and compared directly with existing theoretical models. The backward-propagating indirect noise generated at an orifice plate is shown to be significantly higher than predicted by existing theoretical models for isentropic nozzles.

Key words: acoustics, aeroacoustics

\section{Introduction}

Combustion noise has become a major issue for aero-engine manufacturers. On the one hand, increasingly strict noise emission regulations have prompted efforts to reduce aircraft noise. Significant reductions in jet and turbine noise have made other aircraft noise sources more important. As a result, noise emanating from the combustor is now one of the main contributors to overall aircraft noise, particularly during the landing phase (Dowling \& Mahmoudi 2014). This problem has been 
exacerbated by the introduction of low- $\mathrm{NO}_{x}$ combustors, which burn more unsteadily and are considerably noisier than their predecessors (Dowling \& Stow 2003).

On the other hand, the noise generated inside the combustor may couple with the flame, giving rise to thermoacoustic instabilities. In this scenario, the pressure fluctuations in the combustor may grow uncontrollably, leading to reduced performance, and potentially permanent damage. For these reasons, understanding the underlying mechanisms of combustion noise has become an important topic in academia and industry alike.

Combustion noise can be classified as direct and indirect (Strahle 1971). Direct noise originates from the unsteady heat release associated with the flame, which generates acoustic waves (Bragg 1963). Unsteady heat release also acts as a source of localised temperature perturbations known as 'hot spots' or 'entropy waves', as well as local perturbations of vorticity and composition, all of which are advected with the flow. If there are no flow gradients, linear acoustic, entropic and vortical perturbations do not interact (Chu \& Kovásznay 1958). However, if entropy waves are accelerated/decelerated (as is the case in the exhaust nozzle and turbine blades), they generate acoustic waves, known as 'entropy noise' or indirect noise (Morfey 1973; Ffowcs Williams \& Howe 1975). The acceleration/deceleration of vorticity waves and composition inhomogeneities are also sources of indirect noise (Cumpsty 1979; Magri, O'Brien \& Ihme 2016). Indirect noise propagates both downstream, where it contributes to aircraft noise, and upstream, where it may affect the stability of the system (Polifke, Paschereit \& Klaus 2001; Goh \& Morgans 2013).

Marble \& Candel (1977) provided a simple analytical model for the entropy noise generation mechanism. They considered the case of linear perturbations impinging on a compact nozzle (i.e. for low-frequency perturbations). Starting with the one-dimensional (1-D) linearised Euler equations (LEEs), they derived transfer functions for the acoustic waves generated by an impinging entropy or acoustic wave. Their work has since been built upon and extended to account for different nozzle geometries (Stow, Dowling \& Hynes 2002; Moase, Brear \& Manzie 2007; Goh \& Morgans 2011), as well as nonlinear perturbations (Huet \& Giauque 2013; Huet 2016). Finally, Durán \& Moreau (2013) solved the 1-D LEEs, obtaining results for any given nozzle shape at any frequency.

Experimental investigations of entropy noise have proved to be difficult. Specifically, separating the contributions of direct and indirect noise is particularly arduous as these tend to be highly correlated and hard to distinguish, particularly in the harsh environment of a combustor (Tao et al. 2016). One way to circumvent these difficulties is to carry out a simplified experiment, in which entropy noise is generated artificially using an electric heater rather than a flame. The aim of such an experiment is not to measure combustion noise per se (which in itself is a complex phenomenon involving turbulence, directionality etc.), but rather to measure entropy noise in simplified conditions.

This was attempted by Bohn (1976) and Zukoski \& Auerbach (1976). However, their results were inconclusive due to limitations in the experimental set-up. The entropy wave generator (EWG) rig developed at the German aerospace centre (DLR) overcame these limitations (Bake et al. 2009). Hot spots were generated by pulsing an electric heater, and advected with the flow towards a converging-diverging nozzle (operated both in the subsonic and supersonic regimes).

The DLR EWG experiment generated considerable interest in the research community, leading to several endeavours to explain its results analytically and numerically (Mühlbauer, Noll \& Aigner 2009; Howe 2010; Leyko et al. 2011; 
Giauque, Huet \& Cléro 2012; Durán, Moreau \& Poinsot 2013; Lourier et al. 2014). In the case where the nozzle is operated in the supersonic regime, Leyko et al. (2011) indicated that the measured pressure signal was primarily due to indirect noise. In the subsonic case however, Durán et al. (2013) concluded that the signal was mainly due to the direct noise produced by the electric heater. Conversely, Lourier et al. (2014) argued that the signal could mainly be attributed to indirect noise. Recent work (Becerril et al. 2016) suggests that the contributions of direct and indirect noise in the subsonic case remain unclear. One of the reasons for this uncertainty is related to the modelling of the acoustic reflections at the inlet and outlet of the rig, which greatly affect the simulated results and can lead to almost opposite conclusions.

The original DLR experiment encouraged the development of experiments to investigate the phenomena further. Entropy wave generators similar to the one used at the DLR were developed in Oxford (Hake 2014) and Cambridge (De Domenico, Rolland \& Hochgreb 2017). In our previous experiments at Cambridge, hot spots were generated with an electric heater and accelerated through an orifice plate and the noise was measured upstream of the orifice plate. The results present a clear separation of direct and indirect noise, enabling each to be clearly identified. As with the DLR EWG experiment however, acoustic reflections appear to have a significant effect on the measured pressure signal, making a direct comparison with analytical models impossible, as these are derived for anechoic systems.

The generation of indirect noise requires confinement via a nozzle to accelerate the flow, whilst electrical EWGs can only be operated at low frequencies (producing long wavelengths) owing to physical limitations of the electric heater. This means that in such practical systems, it is difficult, if not impossible, to produce such waves under anechoic conditions, and the resulting pressure measurements appear as a net combination of directly and indirectly generated waves, summed up with the corresponding acoustic reflections arising from the confined environments and boundaries.

The present study provides an analytical, one-dimensional framework to systematically account for the effects of direct and indirect noise and corresponding reflections, such that the results can directly be compared with experiments. Further, once the framework has been validated, it becomes possible to analytically separate the contributions of direct and indirect noise from the model.

The work is organised as follows. In $\S 2$, expressions for the acoustic and entropy waves generated by EWGs are presented. In $\S 3$, the effect of repeated acoustic reflections (reverberation) is analysed, leading to a simple function describing the acoustic pressure inside a reflective EWG system. These expressions are implemented for several limit cases in $\S 4$ and applied to the Cambridge EWG subsonic configuration in $\S 5$. The results of this work are then used to make a direct comparison between the Cambridge EWG experimental results and the direct and indirect noise transfer functions which can be found in the literature.

\section{Waves generated in an entropy wave generator}

The primary function of an entropy wave generator is to produce (often planar) entropy waves, which, once accelerated, generate indirect noise. In the DLR and Cambridge EWGs, entropy waves are produced with an electric heater, which consists of a grid of wires which produce heat pulses via the Joule effect. This heating grid also generates direct noise. Accurate models for both of these acoustic sources are required to identify their individual contributions to the overall signal. 


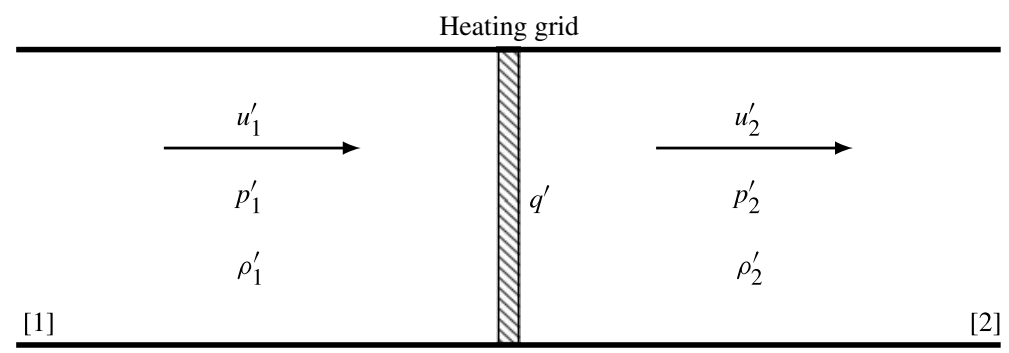

FIgURE 1. Flow perturbations $u^{\prime}, p^{\prime}$ and $\rho^{\prime}$ immediately upstream [1] and downstream

[2] of a compact one-dimensional heating grid.

A model for the direct noise produced by a heating grid is described in Leyko, Nicoud \& Poinsot (2009), Durán et al. (2013). Their model relies on the conservation of mass, enthalpy and entropy across the heating grid. Their analysis is repeated here, using instead conservation of mass, momentum and energy. The indirect noise model derived by Marble \& Candel (1977) is also presented.

\subsection{Direct noise generated by a heating grid}

We consider the one-dimensional case of a heating grid placed in a duct. The grid is considered to be compact; its length is assumed to be negligible compared to the wavelengths of acoustic and entropic perturbations (i.e. low-frequency disturbances), and may be regarded as a discontinuity. Flow variables of interest can be separated into a mean component (denoted with an overbar) and a fluctuating component (denoted with a prime): $\alpha(x, t)=\bar{\alpha}(x)+\alpha^{\prime}(x, t)$. We consider linear perturbations; their amplitude is small relative to the mean quantity (i.e. $\alpha^{\prime} \ll \bar{\alpha}$ ).

In order to capture the effect of the heating grid on the flow variables, jump conditions may be applied, whereby a heat flux $Q$ is added to the flow at the discontinuity. This situation is depicted in figure 1 . In addition, we assume that this flux is small, and that its mean component is negligible $\left(\bar{Q}=0\right.$ and $\left.Q=Q^{\prime}\right)$, so that mean-flow properties are conserved across the discontinuity.

Using the linearised one-dimensional Euler conservation equations for mass, momentum and energy, the flow perturbations on either side of the discontinuity can be related to the added flux $Q^{\prime}$ :

$$
\left.\begin{array}{c}
{\left[\rho^{\prime} \bar{u}+\bar{\rho} u^{\prime}\right]_{1}^{2}=0} \\
{\left[p^{\prime}+\rho^{\prime} \bar{u}^{2}+2 \bar{\rho} \bar{u} u^{\prime}\right]_{1}^{2}=0} \\
{\left[c_{p} \bar{T}\left(\rho^{\prime} \bar{u}+\bar{\rho} u^{\prime}\right)+\bar{\rho} \bar{u}\left(c_{p} T^{\prime}+\bar{u} u^{\prime}\right)\right]_{1}^{2}=Q^{\prime}}
\end{array}\right\}
$$

where $\rho$ is the density, $T$ is the temperature and $u$ is the velocity.

The notation $[\alpha]_{1}^{2}$ denotes the difference between the flow variables immediately upstream [1] and downstream [2] of the discontinuity, such that $[\alpha]_{1}^{2}=\alpha_{2}-\alpha_{1}$.

The flow perturbations in (2.1) can be expressed as a combination of acoustic and entropy waves, both upstream and downstream of the discontinuity, as shown in figure 2 . The acoustic waves $P^{+}$and $P^{-}$propagate upstream and downstream, at speeds $\bar{c}+\bar{u}$ and $\bar{c}-\bar{u}$ respectively, while entropy waves $\sigma$ are advected downstream with the flow speed $\bar{u}$. The amplitudes of these waves can be related to the flow 


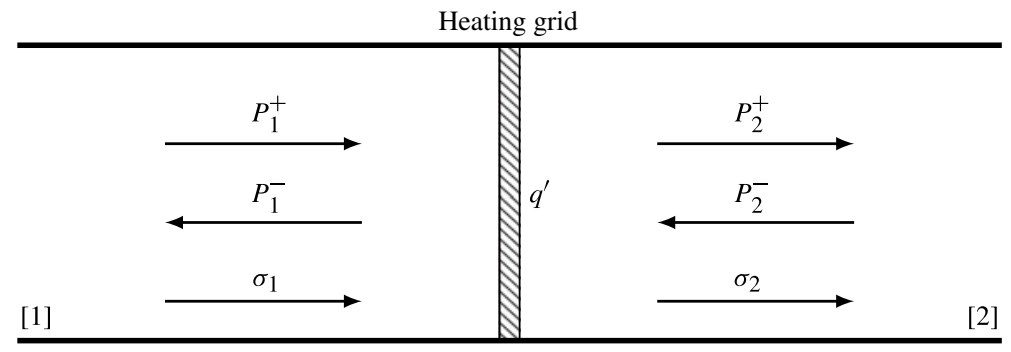

FIGURE 2. Forward and backward acoustic $\left(P^{+}, P^{-}\right)$and entropy waves $\sigma$ upstream [1] and downstream [2] of a compact one-dimensional heating grid.

perturbations:

$$
P^{+} \equiv \frac{1}{2}\left(\frac{p^{\prime}}{\gamma \bar{p}}+\frac{u^{\prime}}{\bar{c}}\right), \quad P^{-} \equiv \frac{1}{2}\left(\frac{p^{\prime}}{\gamma \bar{p}}-\frac{u^{\prime}}{\bar{c}}\right), \quad \sigma \equiv \frac{p^{\prime}}{\gamma \bar{p}}-\frac{\rho^{\prime}}{\bar{\rho}}=\frac{s^{\prime}}{c_{p}}
$$

where $\gamma$ is the heat capacity ratio, $p$ is the pressure, $c$ is the speed of sound and $s$ is the specific entropy.

Combining (2.1) and (2.2) gives jump conditions for the wave amplitudes on either side of the heating grid:

$$
\left.\begin{array}{c}
{\left[\left(1+\frac{1}{\bar{M}}\right) P^{+}+\left(1-\frac{1}{\bar{M}}\right) P^{-}-\sigma\right]_{1}^{2}=0} \\
{\left[\left(1+\frac{1}{\bar{M}}\right)^{2} P^{+}+\left(1-\frac{1}{\bar{M}}\right)^{2} P^{-}-\sigma\right]_{1}^{2}=0} \\
{\left[\left((\gamma-1) \bar{M}+\gamma+\frac{1}{\bar{M}}\right) P^{+}+\left((1-\gamma) \bar{M}+\gamma-\frac{1}{\bar{M}}\right) P^{-}\right]_{1}^{2}=q^{\prime},}
\end{array}\right\}
$$

where $q^{\prime}=Q^{\prime} /\left(\bar{\rho} \bar{u} c_{p} \bar{T}\right)$ is the normalised heat flux change at the interface and $M$ is the Mach number, which is identical upstream and downstream of the heating grid since mean-flow quantities are conserved $\left(\bar{M}_{1}=\bar{M}_{2}\right)$.

In the scenario where there are no incoming waves $\left(P_{1}^{+}=P_{2}^{-}=\sigma_{1}=0\right)$, the heating grid generates forward and backward-propagating acoustic waves $P_{2}^{+}=P_{d}^{+}$and $P_{1}^{-}=$ $P_{d}^{-}$, as well as a forward-propagating entropy wave $\sigma_{2}=\sigma_{d}$. The amplitudes of these waves can be obtained by solving (2.3):

$$
P_{d}^{+}=\frac{1}{2} \frac{\bar{M}}{1+\bar{M}} q^{\prime}, \quad P_{d}^{-}=\frac{1}{2} \frac{\bar{M}}{1-\bar{M}} q^{\prime}, \quad \sigma_{d}=q^{\prime} .
$$

These expressions are identical to those obtained by Leyko et al. (2009) and Durán et al. (2013). Direct noise transfer functions $H_{d}^{-}$and $H_{d}^{+}$may be defined to relate the amplitude these acoustic waves to a given normalised heat release $q^{\prime}$ :

$$
\begin{aligned}
& H_{d}^{-}=\frac{P_{d}^{-}}{q^{\prime}}=\frac{1}{2} \frac{\bar{M}}{1-\bar{M}} \\
& H_{d}^{+}=\frac{P_{d}^{+}}{q^{\prime}}=\frac{1}{2} \frac{\bar{M}}{1+\bar{M}} .
\end{aligned}
$$




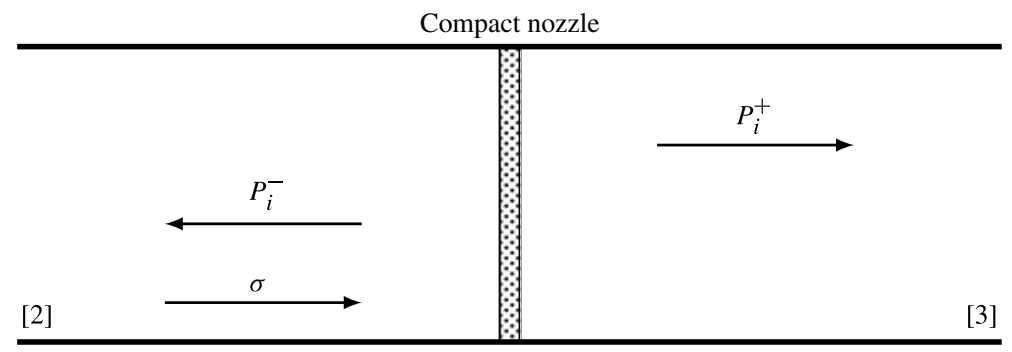

FIgURE 3. Outgoing acoustic waves $\left(P_{i}^{+}, P_{i}^{-}\right)$produced upstream [2] and downstream [3] of a compact nozzle with an impinging entropy wave $\sigma$.

\subsection{Indirect noise generation}

The mechanism by which accelerated entropy waves generate acoustic waves (entropy noise) has been discussed in a number of papers, particularly in the context of nozzles and turbine blade rows (Morfey 1973; Ffowcs Williams \& Howe 1975; Marble \& Candel 1977; Stow et al. 2002; Moase et al. 2007; Huet \& Giauque 2013).

The simplest case is the one considered by Marble \& Candel (1977), in which an entropy wave $\sigma$ impinges upon a compact one-dimensional subsonic or supersonic isentropic nozzle. This gives rise to forward-propagating acoustic wave downstream of the nozzle $P_{i}^{+}$, and a backward-propagating wave upstream $P_{i}^{-}$, as shown in figure 3. The compactness assumption is applicable only in the limit of low-frequency perturbations.

Indirect noise transfer functions $H_{i}^{-}$and $H_{i}^{+}$may be defined to relate the amplitude these acoustic waves to that of the impinging entropy wave $\sigma$. For a subsonic compact isentropic nozzle, they are a function only of the Mach numbers upstream and downstream of the nozzle $\bar{M}_{2}$ and $\bar{M}_{3}$ :

$$
\begin{gathered}
H_{i}^{-}=\frac{P_{i}^{-}}{\sigma}=-\frac{\bar{M}_{3}-\bar{M}_{2}}{1-\bar{M}_{2}} \frac{\frac{1}{2} \bar{M}_{2}}{1+\frac{1}{2}(\gamma-1) \bar{M}_{2} \bar{M}_{3}} \\
H_{i}^{+}=\frac{P_{i}^{+}}{\sigma}=\frac{\bar{M}_{3}-\bar{M}_{2}}{1+\bar{M}_{3}} \frac{\frac{1}{2} \bar{M}_{2}}{1+\frac{1}{2}(\gamma-1) \bar{M}_{2} \bar{M}_{3}} .
\end{gathered}
$$

The indirect noise transfer functions can also be computed at any frequency for noncompact isentropic nozzles (Durán \& Moreau 2013). However, there are currently no transfer functions in the literature for non-isentropic flow elements, such as orifices.

\section{Effect of reflections on the acoustic pressure}

In the case of a one-dimensional duct, the acoustic pressure at any point in time $t$ is obtained by considering the amplitudes of the forward- and backward-propagating acoustic waves at a particular axial location $x$ :

$$
\frac{p^{\prime}}{\gamma \bar{p}}(x, t)=P^{+}(x, t)+P^{-}(x, t) .
$$

When considering the amplitude of direct and indirect noise produced by an entropy wave generator, it may be tempting to use the direct and indirect noise transfer 


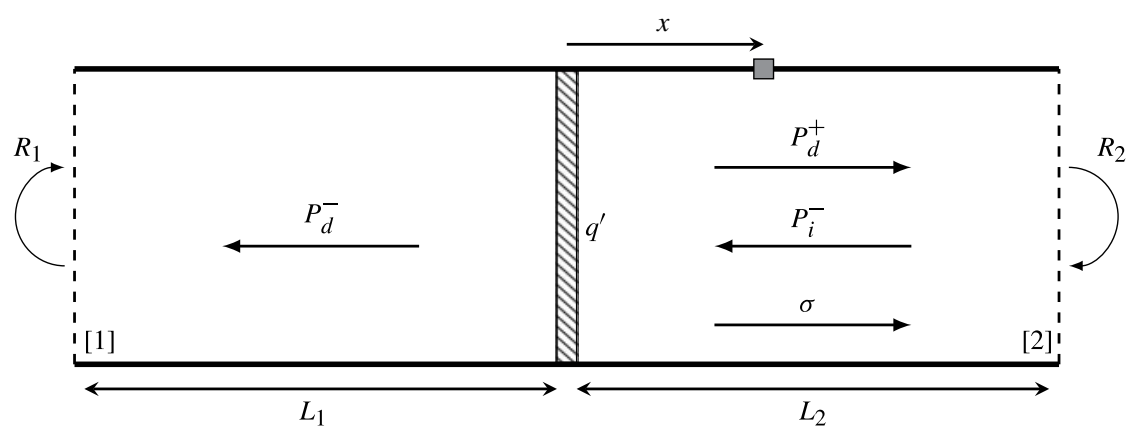

FIGURE 4. Simplified entropy wave generator: acoustic waves are generated by a heating grid (direct noise $P_{d}^{-}$and $P_{d}^{+}$) along with an entropy wave $\sigma$. A backward-propagating acoustic wave is generated at the outlet (indirect noise $P_{i}^{-}$). The acoustic pressure is measured at a distance $x$ from the heating grid.

functions (2.5)-(2.8) in combination with (3.1). This would not always provide a representative result however, as some EWGs present substantial acoustic reflections, which have a strong effect on the acoustic pressure signal. Indeed, acoustic reflections affect the shape of the pressure signal, which modifies the result of a time-domain or harmonic analysis. Furthermore, these reflections may affect direct and indirect noise differently.

The effect of acoustic reflections can be studied by considering the simplified case shown in figure 4, in which a heating grid is placed in a duct terminated with a compact flow restriction (such as a nozzle). Acoustic reflections occur at the inlet and outlet of the system with reflection coefficients $R_{1}$ and $R_{2}$, at distances $L_{1}$ and $L_{2}$ from the grid respectively. The heating grid can be considered as an acoustically compact heat source. There is small change in cross-section at the heating grid location (due to the area occupied by the wires). Since the cross-section is the same upstream and downstream of the heating grid, it can be shown that the heating grid has no effect on the acoustics (since it is acoustically compact). As such, acoustic reflections caused by the heating grid are neglected. We consider the acoustic pressure at a distance $x$ from the heating grid.

The acoustic field can be considered as the superposition of direct noise (generated by the heating grid), and indirect noise (generated at the outlet), such that:

$$
\frac{p^{\prime}}{\gamma \bar{p}}(x, t)=\left.\frac{p^{\prime}}{\gamma \bar{p}}\right|_{d}(x, t)+\left.\frac{p^{\prime}}{\gamma \bar{p}}\right|_{i}(x, t),
$$

where $p^{\prime} /\left.\gamma \bar{p}\right|_{d}$ and $p^{\prime} /\left.\gamma \bar{p}\right|_{i}$ are the acoustic pressures associated with direct and indirect noise respectively. We assume that the heating grid produces a rectangular pulse (i.e. it is activated at $t_{s}=0$ and deactivated at $t=t_{e}$ with no rise time or decay). The entropy wave thus generated is convected the outlet with a convective time delay $\tau_{c}=L_{2} / \bar{u}$, as shown in figure 5. The effects of dispersion and dissipation on the shape of the entropy wave convected downstream are neglected. Direct noise is generated for $t_{s} \leqslant t \leqslant t_{e}$ while indirect noise is generated for $\tau_{c} \leqslant t \leqslant t_{e}+\tau_{c}$.

The effect of acoustic attenuation is neglected for the derivations in $\$ \S 3.1-3.3$ for simplicity. However, it can easily be included in the model, as shown in $\$ 3.4$. 


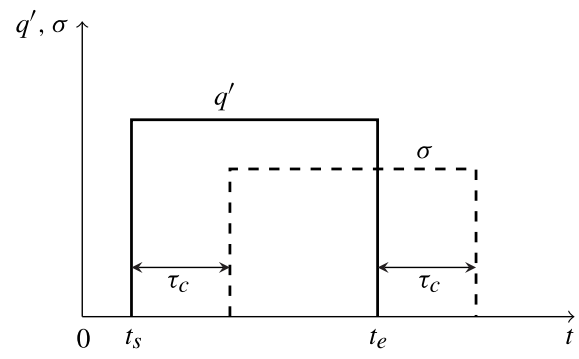

FIGURE 5. Rectangular pulse generated by the entropy generation device (solid line), and rectangular entropy wave at the outlet (dashed line) with a convective time delay $\tau_{c}$.

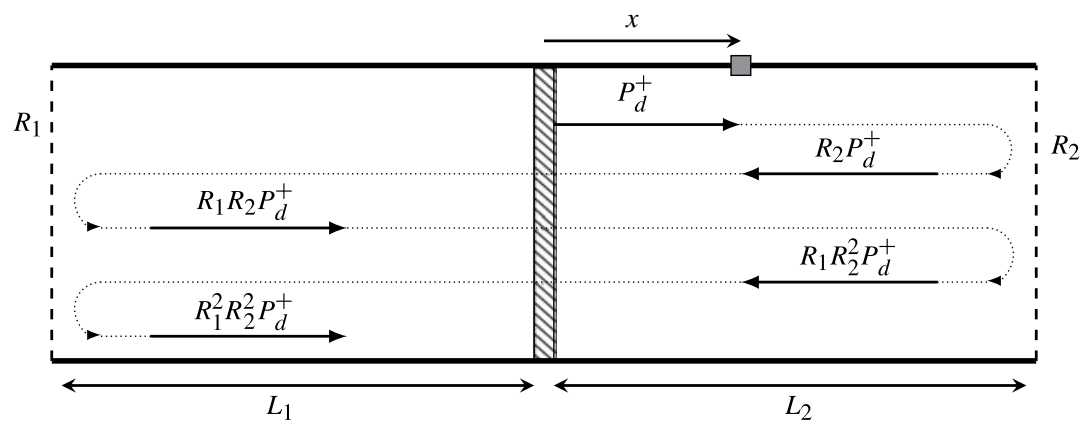

FIGURE 6 . The forward-propagating wave $P_{d}^{+}$generated by the heating grid is successively reflected at the inlet and outlet of the system.

\subsection{Effect of reflections on direct noise}

For the following analysis, we consider acoustic reflections of waves generated during the pulse $\left(0 \leqslant t \leqslant t_{e}\right)$ and after the pulse $\left(t>t_{e}\right)$.

\subsubsection{During the pulse}

When the heating grid is active, acoustic waves $P_{d}^{+}$and $P_{d}^{-}$are continuously generated. At first, the only waves in the duct are those propagating away from the heating grid and their amplitude is given by (2.4). As these waves reach the inlet and outlet of the system, they are reflected, giving rise to waves propagating in the opposite direction, with amplitudes $R_{2} P_{d}^{+}$and $R_{1} P_{d}^{-}$. These reflected waves propagate through the duct, and are once again reflected at the opposite end as $R_{2} R_{1} P_{d}^{-}$and $R_{1} R_{2} P_{d}^{+}$. These successive reflections are shown diagrammatically in figure 6 . As this process repeats itself until the end of the pulse, the overall amplitudes $P^{+}$and $P^{-}$ of the acoustic waves in the duct change and the acoustic pressure inside the system changes accordingly, as shown in (3.1).

Each of the reflected waves appears inside the system after a certain time delay. These time delays are related to the acoustic time scales in the system, i.e. the time taken for an acoustic wave to propagate either forward or backward over a given distance. For example, the time delays of the overall system are obtained by considering the total length $L=L_{1}+L_{2}$ of the system:

$$
\tau^{+}=\frac{L}{\bar{c}+\bar{u}}, \quad \tau^{-}=\frac{L}{\bar{c}-\bar{u}}, \quad T=\tau^{+}+\tau^{-} .
$$


Reflections of $P_{d}^{+}$

\begin{tabular}{lc}
\hline Amplitude & Time delay \\
$P_{d}^{+}$ & $\tau_{x}^{+}$ \\
$R_{2} P_{d}^{+}$ & $\tau_{x}^{+}+T_{2}-T_{x}$ \\
$R_{1} R_{2} P_{d}^{+}$ & $\tau_{x}^{+}+T_{2}+T_{1}$ \\
$R_{1} R_{2}^{2} P_{d}^{+}$ & $\tau_{x}^{+}+2 T_{2}+T_{1}-T_{x}$ \\
$R_{1}^{2} R_{2}^{2} P_{d}^{+}$ & $\tau_{x}^{+}+2 T_{2}+2 T_{1}$
\end{tabular}

Reflections of $P_{d}^{-}$

Amplitude Time delay

$R_{1} P_{d}^{-}$

$\tau_{x}^{+}+T_{1}$

$R_{1} R_{2} P_{d}^{-}$

$\tau_{x}^{+}+T_{1}+T_{2}+T_{x}$

$R_{1}^{2} R_{2} P_{d}^{-}$

$\tau_{x}^{+}+2 T_{1}+T_{2}$

$R_{1}^{2} R_{2}^{2} P_{d}^{-}$

TABLE 1. Amplitudes and time delays associated with reflections of the waves $P_{d}^{+}$ and $P_{d}^{-}$.

These time delays can also be defined for the section upstream of the heating grid, of which the length is $L_{1}$, giving $\tau_{1}^{+}, \tau_{1}^{-}$and $T_{1}^{+}$. Similarly, the time delays corresponding to the section downstream of the heating grid of length $L_{2}$ are $\tau_{2}^{+}, \tau_{2}^{-}$ and $T_{2}^{+}$. Finally, the same definition may be applied for the distance $x$ between the heater location and the pressure measurement location, giving the time delays $\tau_{x}^{+}, \tau_{x}^{-}$ and $T_{x}^{+}$.

The amplitude and time delay associated with each wave passing at the pressure measurement location $x$ is given in table 1 . If we consider that the pulse duration is longer than the acoustic time scale $\left(t_{e}>T\right)$, then the acoustic signal is reverberated, and acoustic reflections occur while the original wave is still being generated.

Combining the information in table 1 with (3.1) enables the acoustic pressure to be obtained at any given point in time. The successive reflections of $P_{d}^{+}$and $P_{d}^{-}$ correspond to the sum of the terms of four geometric series with ratios $R_{1} R_{2}$, and in which each term is separated by a time delay $T=T_{1}+T_{2}$. The general formula for the sum $S_{k}$ of the first $k$ terms of a geometric series is:

$$
S_{k}=\frac{a\left(1-r^{k}\right)}{1-r}
$$

where $r$ is the ratio and $a$ is the first term of the series. This enables us to express the acoustic pressure at the pressure measurement location as:

$$
\begin{aligned}
\left.\frac{p^{\prime}}{\gamma \bar{p}}\right|_{d}\left(x, t \leqslant t_{e}\right)= & \overbrace{P_{d}^{+} \frac{1-\left(R_{1} R_{2}\right)^{\left\lfloor\left(t-\tau_{x}^{+}+T\right) / T\right\rfloor}}{1-R_{1} R_{2}}}^{\text {for } t \geqslant \tau_{x}^{+}}+\overbrace{R_{2} P_{d}^{+} \frac{1-\left(R_{1} R_{2}\right)^{\left\lfloor\left(t+\tau_{x}^{-}+T_{1}\right) / T\right\rfloor}}{1-R_{1} R_{2}}}^{\text {for } t \geqslant T_{2}-\tau_{x}^{-}} \\
& +\underbrace{R_{1} P_{d}^{-} \frac{1-\left(R_{1} R_{2}\right)^{\left\lfloor\left(t-\tau_{x}^{+}+T_{2}\right) / T\right\rfloor}}{1-R_{1} R_{2}}}_{\text {for } t \geqslant \tau_{x}^{+}+T_{1}}+\underbrace{R_{1} R_{2} P_{d}^{-} \frac{1-\left(R_{1} R_{2}\right)^{\left\lfloor\left(t+\tau_{x}^{-}\right) / T\right\rfloor}}{1-R_{1} R_{2}}}_{\text {for } t \geqslant T_{1}+T_{2}+\tau_{x}^{-}} .
\end{aligned}
$$

In a real system, we have $\left|R_{1} R_{2}\right|<1$, and (3.5) converges. If the acoustic time scale $T$ is significantly shorter than the pulse duration $t_{e}$, then the acoustic pressure will quickly converge towards:

$$
\left.\lim _{t \rightarrow \infty} \frac{p^{\prime}}{\gamma \bar{p}}\right|_{d}=\frac{\left(R_{1} P_{d}^{-}+P_{d}^{+}\right)\left(1+R_{2}\right)}{1-R_{1} R_{2}} .
$$




\begin{tabular}{lcccc}
\multicolumn{2}{c}{ Reflections of $P_{D}$ (forward) } & & Reflections of $P_{D}$ (backward) \\
\cline { 1 - 2 } \cline { 5 - 5 } Amplitude & Time delay & & Amplitude & Time delay \\
$P_{D}$ & $t_{e}$ & & $P_{D}$ & $t_{e}$ \\
$R_{2} P_{D}$ & $t_{e}+T_{2}-T_{x}$ & & $R_{1} P_{D}$ & $t_{e}+T_{1}+T_{x}$ \\
$R_{1} R_{2} P_{D}$ & $t_{e}+T_{2}+T_{1}$ & & $R_{1} R_{2} P_{D}$ & $t_{e}+T_{1}+T_{2}$ \\
$R_{1} R_{2}^{2} P_{D}$ & $t_{e}+2 T_{2}+T_{1}-T_{x}$ & & $R_{1}^{2} R_{2} P_{D}$ & $t_{e}+2 T_{1}+T_{2}+T_{x}$ \\
$R_{1}^{2} R_{2}^{2} P_{D}$ & $t_{e}+2 T_{2}+2 T_{1}$ & & $R_{1}^{2} R_{2}^{2} P_{D}$ & $t_{e}+2 T_{1}+2 T_{2}$ \\
\multicolumn{1}{c}{$\vdots$} & $\vdots$ & $\vdots$ & $\vdots$
\end{tabular}

TABLE 2. Amplitudes and time delays associated with reflections of the forward and backward waves $P_{D}$.

\subsubsection{After the pulse}

Once the heating grid is deactivated at $t=t_{e}$, it no longer generates acoustic waves. The only contributions to the acoustic pressure come from pre-existing waves propagating and reflecting at the boundaries of the system.

The acoustic pressure immediately after the heating grid is deactivated is $p^{\prime} /\left.\gamma \bar{p}\right|_{d}\left(x, t_{e}\right)$. We make the simplifying assumption that the acoustic field is comprised of a forward- and a backward-propagating wave of equal amplitude $P_{D}$. The wave amplitude $P_{D}$ must satisfy (3.1) and can be assumed to be:

$$
P_{D}=\left.\frac{1}{2} \frac{p^{\prime}}{\gamma \bar{p}}\right|_{d}\left(x, t_{e}\right) \text {. }
$$

The amplitude and time delay associated with each wave passing at the pressure measurement location is given in table 2 .

Combining the information in table 2 with (3.1) enables the acoustic pressure to be obtained at any given point in time. The successive reflections of $P_{D}$ correspond to the terms of four geometric sequences with ratios $R_{1} R_{2}$, and in which each term is separated by a time delay $T$. The acoustic pressure in the tube can be approximated as the successive terms of two of these four sequences, and expressed as:

$$
\left.\frac{p^{\prime}}{\gamma \bar{p}}\right|_{d}\left(t>t_{e}\right)=P_{D}\left(R_{1} R_{2}\right)^{\left\lfloor\left(t-t_{e}\right) / T\right\rfloor}+R_{2} P_{D}\left(R_{1} R_{2}\right)^{\left\lfloor\left(t-t_{e}-T_{1}-T_{x}+T\right) / T\right\rfloor} .
$$

The accuracy of this approximation is examined in limit case IV (§4).

\subsection{Effect of reflections on indirect noise}

The analysis carried out for direct noise can be replicated for indirect noise. In this case, indirect noise manifests itself as a single backward-propagating acoustic wave $P_{i}^{-}$generated at the outlet (we assume that the downstream-propagating component $P_{i}^{+}$ is not reflected back into the system). We consider acoustic reflections while indirect noise is being generated $\left(\tau_{c} \leqslant t \leqslant t_{e}+\tau_{c}\right)$, and afterwards $\left(t>t_{e}+\tau_{c}\right)$.

\subsubsection{During indirect noise generation}

As with direct noise, the acoustic wave associated with indirect noise is reflected at the inlet and outlet repeatedly. Each reflection has an associated acoustic time delay, 


\begin{tabular}{lc} 
& Reflections of $P_{i}^{-}$ \\
\hline Amplitude & Time delay \\
$P_{i}^{-}$ & $\tau_{c}+\tau_{2}^{-}-\tau_{x}^{-}$ \\
$R_{1} P_{i}^{-}$ & $\tau_{c}+\tau_{2}^{-}-\tau_{x}^{-}+T_{1}+T_{x}$ \\
$R_{1} R_{2} P_{i}^{-}$ & $\tau_{c}+\tau_{2}^{-}-\tau_{x}^{-}+T_{1}+T_{2}$ \\
$R_{1}^{2} R_{2} P_{i}^{-}$ & $\tau_{c}+\tau_{2}^{-}-\tau_{x}^{-}+2 T_{1}+T_{x}+T_{2}$ \\
$R_{1}^{2} R_{2}^{2} P_{i}^{-}$ & $\tau_{c}+\tau_{2}^{-}-\tau_{x}^{-}+2 T_{1}+2 T_{2}$ \\
$\quad \vdots$ & $\vdots$
\end{tabular}

TABLE 3. Amplitudes and time delays associated with reflections of the indirect noise wave $P_{i}^{-}$.

as shown in table 3. This enables us to express the acoustic pressure associated with indirect noise at the measurement location as:

$$
\begin{aligned}
\left.\frac{p^{\prime}}{\gamma \bar{p}}\right|_{i}\left(x, t \leqslant t_{e}+\tau_{c}\right)= & \overbrace{P_{i}^{-}}^{\frac{1-\left(R_{1} R_{2}\right)^{\left\lfloor\left(t-\tau_{c}-\tau_{2}^{-}+\tau_{x}^{-}+T\right) / T\right\rfloor}}{1-R_{1} R_{2}}} \\
& +\underbrace{R_{1} P_{i}^{-} \frac{1-\left(R_{1} R_{2}\right)^{\left\lfloor\left(t-\tau_{c}+\tau_{2}^{+}-\tau_{x}^{+}\right) / T\right\rfloor}}{1-R_{1} R_{2}}}_{\text {for } t \geqslant \tau_{c}+\tau_{2}^{-}+\tau_{x}^{+}+T_{1}},
\end{aligned}
$$

which will converge towards:

$$
\left.\lim _{t \rightarrow \infty} \frac{p^{\prime}}{\gamma \bar{p}}\right|_{i}=\frac{P_{i}^{-}\left(1+R_{1}\right)}{1-R_{1} R_{2}}
$$

\subsubsection{After indirect noise generation}

Once indirect noise is no longer being generated, the only acoustic waves in the duct are pre-existing waves propagating and reflecting at the boundaries of the system. As in $\$ 3.1 .2$, we assume that the acoustic field is comprised of a forward- and a backward-propagating wave of equal amplitude. Their amplitude $P_{I}$ must satisfy (3.1) and can be assumed to be:

$$
P_{I}=\left.\frac{1}{2} \frac{p^{\prime}}{\gamma \bar{p}}\right|_{i}\left(x, t_{e}+\tau_{c}\right)
$$

Once again, the acoustic pressure can be written as the successive terms of two geometric sequences:

$$
\left.\frac{p^{\prime}}{\gamma \bar{p}}\right|_{i}\left(x, t>t_{e}+\tau_{c}\right)=P_{I}\left(R_{1} R_{2}\right)^{\left\lfloor\left(t-t_{e}-\tau_{c}\right) / T\right\rfloor}+R_{2} P_{I}\left(R_{1} R_{2}\right)^{\left\lfloor\left(t-t_{e}-\tau_{c}-T_{1}-T_{x}+T\right) / T\right\rfloor} .
$$




\subsection{Simplified expressions}

If we consider that the pulse duration is much longer than the acoustic time scale $\left(t_{e} \gg T\right)$, then (3.5), (3.8), (3.9) and (3.12) may be approximated to obtain:

$$
\begin{aligned}
\left.\frac{p^{\prime}}{\gamma \bar{p}}\right|_{d}(x, t) \approx \begin{cases}\left(P_{d}^{+}+P_{d}^{-} R_{1}\right)\left(1+R_{2}\right) \frac{1-\left(R_{1} R_{2}\right)^{\lfloor(t+T) / T\rfloor}}{1-R_{1} R_{2}} & \text { for } t \in\left[0, t_{e}\right] \\
2 P_{D}\left(R_{1} R_{2}\right)^{\left\lfloor\left(t-t_{e}+T\right) / T\right\rfloor} & \text { for } t \in] t_{e},+\infty[\end{cases} \\
\left.\frac{p^{\prime}}{\gamma \bar{p}}\right|_{i}(x, t) \approx \begin{cases}P_{i}^{-}\left(1+R_{1}\right) \frac{1-\left(R_{1} R_{2}\right)^{\left\lfloor\left(t-\tau_{c}+T\right) / T\right\rfloor}}{1-R_{1} R_{2}} & \text { for } t \in\left[\tau_{c}, t_{e}+\tau_{c}\right] \\
2 P_{I}\left(R_{1} R_{2}\right)^{\left\lfloor\left(t-t_{e}-\tau_{c}+T\right) / T\right\rfloor} & \text { for } t \in] t_{e}+\tau_{c},+\infty[,\end{cases}
\end{aligned}
$$

where the wave amplitudes $P_{d}^{-}, P_{d}^{+}$and $P_{i}^{-}$can be computed from suitable transfer functions, such as those presented in (2.5)-(2.7).

Equations (3.13) and (3.14) are straightforward expressions for the evolution of acoustic pressure due to the successive reflections of direct and indirect noise respectively. These show that the pressure in the duct closely follows a power law, governed by the acoustic time scale of the system $T$ and the product of the inlet and outlet reflection coefficients $R_{1} R_{2}$.

In their work, Leyko et al. (2009) and Durán et al. (2013) define an indirect to direct noise ratio $\eta$ by considering the acoustic waves brought about by the direct and indirect noise mechanisms ( $w_{d}$ and $w_{i}$ respectively). In our case, the indirect noise wave corresponds to $w_{i}=P_{i}^{-}$. The direct noise wave $w_{d}$ however, must capture the effect of both $P_{d}^{+}$and $P_{d}^{-}$. Since we are interested in what occurs downstream of the heating grid, we take $w_{d}=P_{d}^{+}+R_{1} P_{d}^{-}$, capturing the fact that the backward wave $P_{d}^{-}$must be reflected at the inlet before it is measured by a pressure transducer downstream of the heating grid with an amplitude $R_{1} P_{d}^{-}$. As such, we define:

$$
\eta=\frac{w_{i}}{w_{d}}=\frac{P_{i}^{-}}{P_{d}^{+}+R_{1} P_{d}^{-}} .
$$

An analytical expression for $\eta$ can be obtained using (2.5)-(2.7). Another way to gauge the ratio of indirect to direct noise is to compare their contributions $p_{d}^{\prime}$ and $p_{i}^{\prime}$ to the total acoustic pressure $p^{\prime}$ directly. This can only be done when these contributions can be clearly identified. Under the conditions stated in $\S 3$ (reverberation with $t_{e} \gg T$, no attenuation, rectangular acoustic pulse), then this ratio can be computed from (3.6) and (3.10) as:

$$
\varphi=\frac{p_{i}^{\prime}}{p_{d}^{\prime}}=\frac{P_{i}^{-}\left(1+R_{1}\right)}{\left(P_{d}^{+}+R_{1} P_{d}^{-}\right)\left(1+R_{2}\right)}=\frac{1+R_{1}}{1+R_{2}} \eta .
$$

These two definitions for the indirect to direct noise ratio are equivalent only if $R_{1}=R_{2}$. This highlights the importance of taking reflection coefficients into account when considering the noise ratio in a real system with acoustic reflections. Indeed, using $\eta$ to predict the ratio of indirect to direct noise $\varphi$ in a pressure signal would lead to the wrong result if $R_{1} \neq R_{2}$. This distinction is particularly relevant when comparing experimental results to simulations or analytical expressions. 


\subsection{Effect of acoustic attenuation}

Acoustic attenuation occurs in the tube due to viscous effects, as well as losses at the walls. The amplitude change of a acoustic wave due to attenuation can be expressed as:

$$
P(l)=P_{0} \mathrm{e}^{-\alpha l},
$$

where $P_{0}$ is the original amplitude of the wave, $P(l)$ is the amplitude of the wave at a distance $l$ from its origin and $\alpha$ is the attenuation coefficient. The effect of acoustic attenuation can be included in the results presented in $\S \S 3.1-3.3$. Consider the case of a wave $P_{d}^{+}$generated at the heating grid. If acoustic attenuation is neglected, the wave propagates towards the outlet and returns with an amplitude $P_{d}^{+} R_{2}$. If acoustic attenuation is taken into account, the wave is attenuated over a distance $2 L_{2}$, and returns with an amplitude $P_{d}^{+} R_{2} \mathrm{e}^{-2 \alpha L_{2}}$.

Including attenuation terms of the form $\mathrm{e}^{-\alpha l}$ throughout the derivation in $\S \S 3.1-$ 3.3 yields modified expressions for the acoustic pressure due to reverberation. These modified expressions can be easily obtained by substituting $R_{1}$ with $R_{1} e^{-2 \alpha\left(L_{1}+x\right)}$ and $R_{2}$ by $R_{2} e^{-2 \alpha\left(L_{2}-x\right)}$ in (3.5)-(3.16). For example, the simplified expression for direct noise becomes:

$$
\left.\frac{p^{\prime}}{\gamma \bar{p}}\right|_{d}(x, t) \approx\left\{\begin{array}{cl}
\left(P_{d}^{+}+P_{d}^{-} R_{1} \mathrm{e}^{-2 \alpha\left(L_{1}+x\right)}\right)\left(1+R_{2} \mathrm{e}^{-2 \alpha\left(L_{2}-x\right)}\right) & \\
\times \frac{1-\left(R_{1} R_{2} \mathrm{e}^{-2 \alpha L}\right)^{\lfloor(t+T) / T\rfloor}}{1-R_{1} R_{2} \mathrm{e}^{-2 \alpha L}} & \text { for } t \in\left[0, t_{e}\right] \\
2 P_{D}\left(R_{1} R_{2} \mathrm{e}^{-2 \alpha L}\right)^{\left\lfloor\left(t-t_{e}+T\right) / T\right\rfloor} & \text { for } t \in] t_{e},+\infty[.
\end{array}\right.
$$

\subsection{Analytical method for non-rectangular pulses}

One of the limitations of the reverberation model outlined above is that it requires the acoustic forcing signal to be rectangular. In real EWGs, this is not usually the case. However, the acoustic pressure resulting from the reverberation of non-rectangular acoustic signals can be closely approximated analytically by discretising the acoustic signal.

Indeed, an acoustic signal of arbitrary shape can be approximated as the succession of rectangular pulses of varying amplitudes and of width $\delta t$. As $\delta t$ is decreased, the approximation of the arbitrary signal becomes increasingly accurate. In this sense, any acoustic signal can be closely approximated as a train of several successive rectangular acoustic waves. This is shown in figure 7 in the case where the acoustic pulse is a raised sine wave (of the form $\sin ^{2}(\omega t)$ ) lasting $t_{e}=200 \mathrm{~ms}$ with an amplitude $P^{+}=10^{-5}$. The acoustic wave is shown in $(a)$, and rectangular approximations for $\delta t=20 \mathrm{~ms}$ and $\delta t=10 \mathrm{~ms}$ are shown in $(b)$ and $(c)$ respectively.

The acoustic pressure resulting from the reverberation of the non-rectangular signal can then be estimated as the sum of the acoustic pressures due to each of the rectangular waves used to construct it. This can be calculated analytically by using the reverberation model presented in (3.13) and (3.14). In principle, the accuracy of this approximation can be improved by decreasing $\delta t$ as required. Note that if the rectangular pulse width $\delta t$ is smaller than the acoustic time scale $T$, the 'floored' terms $\lfloor\cdot\rfloor$ must be replaced by $(\cdot)$ to recover a physical solution. 


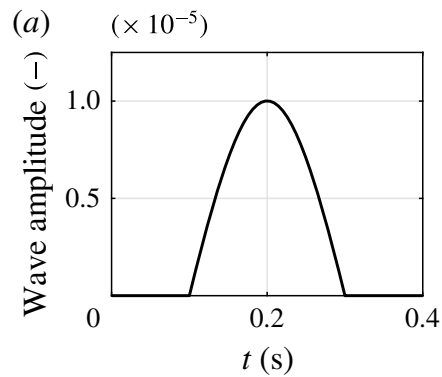

(b)

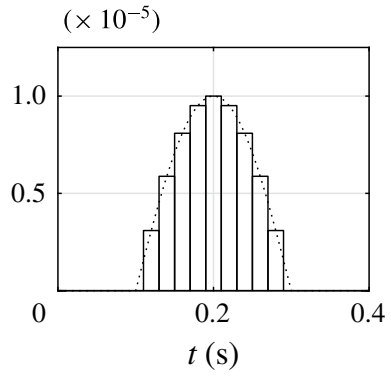

(c)

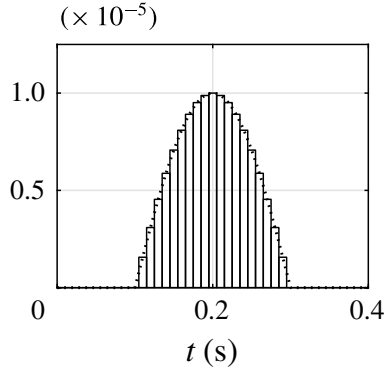

FIGURE 7. (a) Non-rectangular acoustic wave, $(b)$ non-rectangular acoustic wave (dotted line) and rectangular approximation for $\delta t=20 \mathrm{~ms}$, $(c)$ non-rectangular acoustic wave (dotted line) and rectangular approximation for $\delta t=10 \mathrm{~ms}$.

\section{Limit cases}

The expressions derived in $\S 3$ can be used to compute the acoustic pressure in an EWG. While these expressions assume ideal conditions (one-dimensional system, localised reflections), they may be used to show some of the limit cases for this type of system.

This section presents eight limit cases showcasing the range of behaviours that may be displayed by EWGs. Cases I-IV examine the influence of acoustic reflections on direct noise, while cases V-VII examine the interaction between direct and indirect noise. Case VIII corresponds to the case where the forcing acoustic pulse is not rectangular. All cases correspond to the geometry shown in figure 4, where the lengths upstream and downstream of the heating grid are $L_{1}=1 \mathrm{~m}$ and $L_{2}=2 \mathrm{~m}$ respectively. The pressure sensor is located $x=1 \mathrm{~m}$ downstream of the heating grid. Acoustic reflections occur at the inlet and outlet of the duct (specified by $R_{1}$ and $R_{2}$ respectively). Acoustic attenuation is neglected $(\alpha=0)$.

The expressions derived in this paper assume low-frequency perturbations, which in this case corresponds to $t_{e} \gg T$, i.e. the pulse is much longer than the acoustic time scale. To place ourselves in this scenario, we take $t_{e}=200 \mathrm{~ms}$ for all cases, with $T \approx 17.5 \mathrm{~ms}$ depending on the case (the sound speed is taken as $c=345 \mathrm{~m} \mathrm{~s}^{-1}$ ). These values are broadly representative of those in the DLR and Cambridge EWG experiments.

As a point of comparison, the acoustic pressure for cases I-VIII is also computed numerically using a low-order model. Here, OSCILOS is used, which simulates 1-D wave propagation and wave reflection based on the linearised Euler equations. (OSCILOS is an open source code for simulating combustion instability. It is being developed by Dr A. Morgans and co-workers at the Department of Aeronautics, Imperial College London, UK.)

\subsection{Cases I-IV: direct noise}

In cases I-IV, we consider that there is no indirect noise generation at the outlet, meaning that only direct noise is generated $\left(P_{i}^{-}=0\right)$. For simplicity, the forcing acoustic pulse is considered to be rectangular, with $P_{d}^{+}=P_{d}^{-}=10^{-5}$ (neglecting the small mean-flow effect shown in (2.4)). The reflection coefficients used for each case are listed in table 4. The reflection coefficients are chosen to cover a range of limit behaviours. The acoustic pressure histories are shown for cases I-IV in figure 8 . 


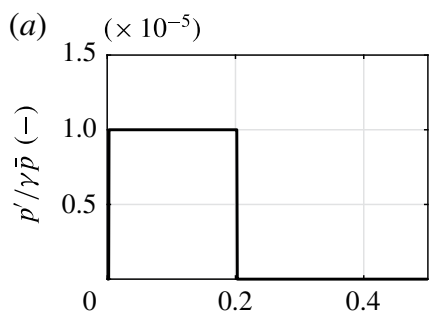

(b)

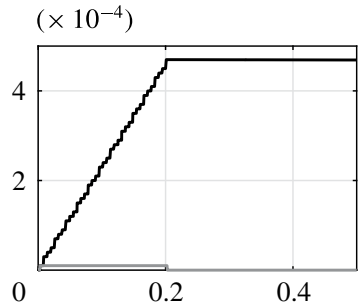

$(d)$

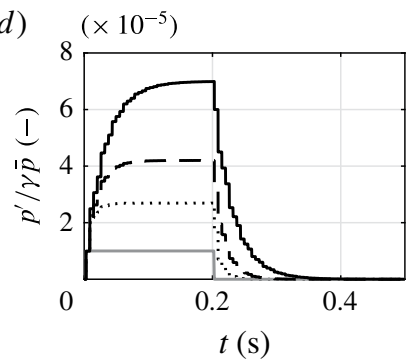

(e)

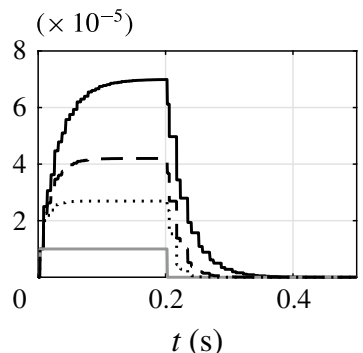

(c) $\left(\times 10^{-5}\right)$
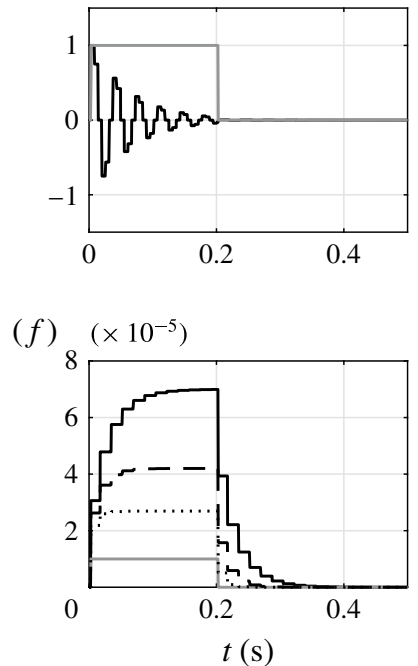

FIGURE 8. Non-dimensionalised acoustic pressure history $p^{\prime} / \gamma \bar{p}$ at $x=1 \mathrm{~m}$ (solid black line) and case I results (for reference) (solid grey line). (a) Case I: anechoic, $(b)$ case II: fully reflective, $(c)$ case III: open ended, $(d)$ case IV: partial reflections (OSCILOS) for $R_{2}=0.25$ (dotted line), $R_{2}=0.5$ (dashed line) and $R_{2}=0.75$ (solid line), $(e)$ case IV: partial reflections (full analytical expressions), $(f)$ case IV: partial reflections (simplified analytical expression).

$\begin{array}{lccc}\text { Limit case } & \text { Description } & R_{1} & R_{2} \\ \text { I } & \text { Anechoic } & 0 & 0 \\ \text { II } & \text { Fully reflective } & 0.99 & 1 \\ \text { III } & \text { Open-ended } & 0.75 & -1 \\ \text { IV } & \text { Partial reflections } & 0.75 & 0.25 \rightarrow 0.75\end{array}$

TABLE 4. Description of limit cases I-IV with corresponding reflection coefficients $R_{1}$ and $R_{2}$.

In case $\mathrm{I}$, the system is anechoic $\left(R_{1}=R_{2}=0\right)$. As a result, the only contribution to the acoustic pressure at the pressure measurement location $x=1 \mathrm{~m}$ is $P_{d}^{+}$. The acoustic pressure $p^{\prime} / \gamma \bar{p}(x, t)$ rises sharply to a maximum value of $P_{d}^{+}=10^{-5}$ at $t=\tau_{x}^{+}$, and falls back to 0 at $t=t_{e}+\tau_{x}^{+}$. As expected, this corresponds to a rectangular pulse (identical to the forcing pulse), with a time shift $\tau_{x}^{+}$due to the acoustic propagation time delay between the heating grid and the pressure measurement location.

A fully reflective system corresponds to $R_{1}=R_{2}=1$, but the expressions derived in $\S 3$ are not defined for $R_{1} R_{2}=1$. A virtually identical result is obtained in case II by taking $R_{1}=0.99$ and $R_{2}=1$. In this scenario, waves are (nearly) fully reflected at the boundaries of the system, and there are effectively no acoustic losses. Given that waves $P_{d}^{-}$and $P_{d}^{+}$are being continuously generated for $t \leqslant t_{e}$, acoustic energy accumulates inside the system as acoustic waves repeatedly reflect at the boundaries with the same sign. As a result, the acoustic pressure rises gradually to reach a large value (approximately 45 times larger than in the anechoic case here). 
Paying close attention to the acoustic pressure history reveals that the pressure does not rise continuously, but instead increases 'step by step'. Each 'step' corresponds to a wave being reflected at a boundary system and 'adding' to the total acoustic pressure. These steps are separated by the acoustic time scale $T$, which is the time taken for a given acoustic wave to be reflected back to its original position. The 'staircase' aspect of the computed signal is not related to a numerical method, but is instead due to the fact that the pulse is perfectly rectangular. In a real situation, the forcing pulse is smoother, and a combination of fluid mechanical effects (primarily diffusion) make this aspect less pronounced. After the end of the pulse (once the heating grid is switched off), acoustic energy is conserved as there are no acoustic losses in the system and the pressure stays constant.

In case III, the inlet of the system is partially reflective $\left(R_{1}=0.5\right)$ and the outlet is open ended $\left(R_{2}=-1\right)$. This means that acoustic waves propagating downstream are reflected with a negative sign; instead of increasing the total acoustic pressure as in case II, these reflected waves decrease the acoustic pressure and 'cancel out' incoming waves. The acoustic pressure oscillates around zero, bounded between $P_{d}$ and $-P_{d}$. The amplitude of these oscillations decreases with time. This is due to the partial reflections at the inlet, which make each successively reflected wave slightly smaller in amplitude that the previous one. Once the forcing pulse ends, the acoustic pressure quickly returns to zero. This behaviour is similar to case A in De Domenico et al. (2017).

The results for case IV are shown in figure 8 for $(d)$ the low-order model, $(e)$ the analytical expressions (3.5) and (3.8) and $(f)$ the simplified analytical expressions (3.13). Case IV corresponds to the case where both the inlet and outlet of the system are partially reflective. As in case II, waves are positively reflected, leading to an overall increase of the acoustic pressure, but as shown in case III, the amplitudes of the partially reflected waves at the inlet decrease as time goes on.

As a result, the acoustic pressure converges towards a maximum value, which is consistent with (3.6). For all values of $R_{2}$, the maximum acoustic pressure is higher than in case I (anechoic), but lower than in case II (fully reflective). Notably, as $R_{2}$ is increased from $0.25-0.75$, the acoustic pressure increases. The results given by OSCILOS $(d)$ and the analytical expressions $(e)$ are identical. The simplified expressions however, show a coarser signal due to the fact that small time delays are neglected. However, the signal converges towards the same maximum, and the overall growth rate (neglecting the slightly different step changes) is the same.

Once the heating grid is switched off at $t=200 \mathrm{~ms}$, the acoustic pressure decays as acoustic energy leaves the system. Each discontinuous decrease corresponds to an acoustic wave reaching a boundary, at which point it loses a fraction $\left(1-R_{1}\right.$ or $\left.1-R_{2}\right)$ of its amplitude. Here, the analytical results (figure $8 e, f$ ) give a coarser result than the OSCILOS simulations (figure $8 d$ ). This is because of the simplifying assumptions made in \$3.1.2. Most notably, we have approximated the successive terms of four series as the terms of just two series, which explains why the pressure decays in larger steps in the analytical results. However, the decay rate in all cases is exactly the same (e.g. the acoustic pressure falls to $10 \%$ of its maximum at $t=273 \mathrm{~ms}$ in all cases).

\subsection{Cases V-VII: direct and indirect noise}

Cases V-VII highlight the behaviours of both direct and indirect noise. Here, we assume that there is flow acceleration downstream of the heating grid location such that indirect noise is generated at the outlet. We consider rectangular pulses with 

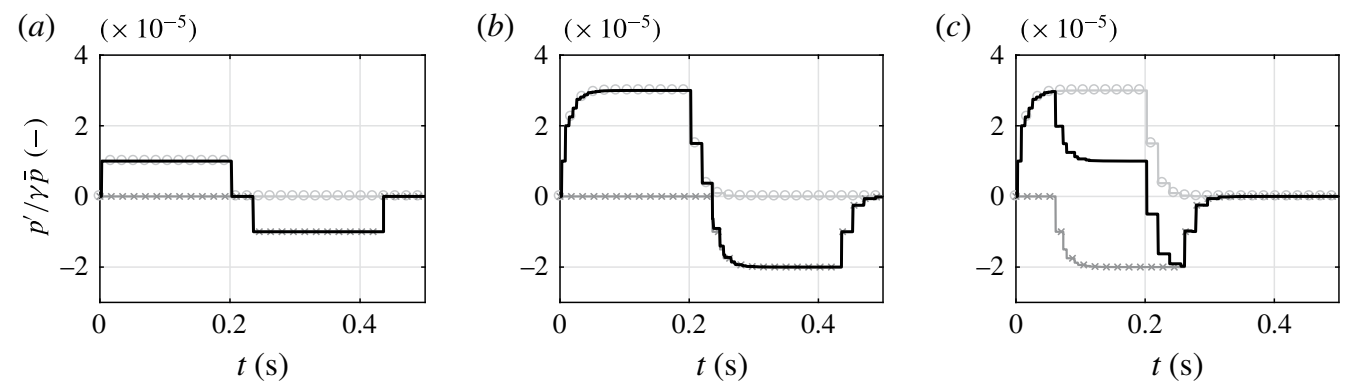

FIGURE 9. Non-dimensionalised acoustic pressure history $p^{\prime} / \gamma \bar{p}$ at $x=1 \mathrm{~m}$ of total noise (solid line), direct noise (light grey solid line with circles) and indirect noise (dark grey solid line with crosses). (a) Case V: anechoic (low Mach), (b) case VII: partial reflections (low Mach), (c) case VII: partial reflections.

$\begin{array}{lcccc}\text { Limit case } & \text { Description } & R_{1} & R_{2} & M \\ \text { V } & \text { Anechoic } & 0 & 0 & 0.025 \\ \text { VI } & \text { Partial reflections } & 0.5 & 0.5 & 0.025 \\ \text { VII } & \text { Partial reflections (high Mach) } & 0.5 & 0.5 & 0.1\end{array}$

TABLE 5. Description of limit cases V-VII with corresponding reflection coefficients $R_{1}$ and $R_{2}$ and Mach number $M$.

$P_{d}^{+}=P_{d}^{-}=10^{-5}$ and $P_{i}^{-}=-10^{-5}$ in all cases regardless of the flow conditions. In a real system, these values would vary based on the flow, but using a constant value enables different cases to be compared on an even basis. For each case we vary the reflection coefficients, as well as the Mach number in the duct $M$ (which primarily affects the convective time delay $\tau_{c}$ ). These parameters are shown in table 5 . The acoustic pressure histories for cases V-VII are shown in figure 9.

In case $\mathrm{V}$, the Mach number is relatively low, and there is a large convective time delay $\tau_{c}$ between the times at which at which direct and indirect noise are generated. In this case, we have $\tau_{c}>t_{e}$ meaning that entropy waves reach the outlet once direct noise is no longer being generated. This can be seen in the acoustic pressure histories, which shows a very clear temporal separation between direct and indirect noise. As in case I, the direct noise appears as a rectangular pulse of duration $t_{e}$ with a maximum value of $P_{d}=10^{-5}$. The indirect noise is also a rectangular pulse, but time shifted by $\tau_{c}$, with a maximum of $P_{i}$. The total acoustic pressure in the tube and shows a positive pulse (corresponding to the direct noise), followed by a negative pulse (corresponding to entropy noise).

Case VI is identical to case V except that the Mach number in the system is four times larger (and the convective time delay $\tau_{c}$ is four times smaller as a result). We now have $\tau_{c}<t_{e}$, meaning that there is an overlap between the generation of direct and indirect noise. Once again, the total acoustic pressure corresponds to a positive pulse followed by a negative pulse. In this case however, direct and indirect noise interact de-constructively, and the duration of these pulses is reduced to $\tau_{c}$. At the limit where the convection and acoustic time scales $\tau_{c}$ and $T$ are 0 (i.e. there is no separation between the entropy wave source and the outlet), then direct and indirect noise cancel each other out completely. 

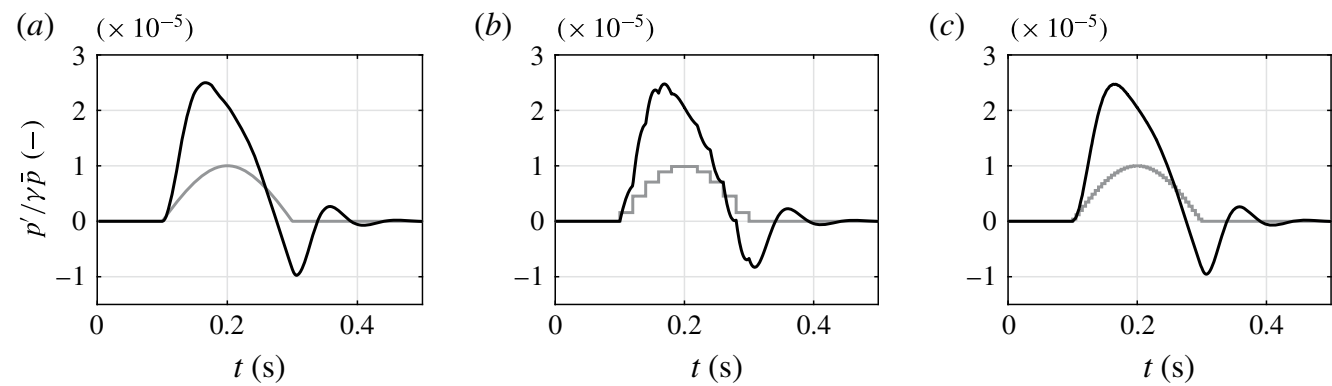

FIGURE 10. Non-dimensionalised acoustic pressure history $p^{\prime} / \gamma \bar{p}$ at $x=1 \mathrm{~m}$ for case VIII (solid black line) with acoustic forcing signal (solid grey line) obtained with (a) OSCILOS, (b) analytical method for $\delta t=20 \mathrm{~ms}$ and (c) analytical method for $\delta t=5 \mathrm{~ms}$.

Case VI is a combination of cases IV and V, where direct and indirect noise are clearly separated, and there are partial reflections at the inlet and outlet of the system. As expected, direct and indirect noise are effectively amplified compared to case $\mathrm{V}$ (anechoic). Direct and indirect noise are not amplified by the same factor however: while both of them give a maximum pressure amplitude of $10^{-5}$ in the anechoic case, they reach $3 \times 10^{-5}$ and $2 \times 10^{-5}$ respectively. This can be explained by the fact that indirect noise is produced directly at the outlet, unlike direct noise. This difference in amplification is made apparent in (3.16).

Case VII is similar to case VI, except that there is now a large overlap between direct and indirect noise due to the decreased convective time delay. This overlap results in a merging effect, whereby direct and indirect noise interact de-constructively and the overall acoustic pressure is reduced.

\subsection{Case VIII: non-rectangular acoustic pulse with complex reflection coefficient}

For case VIII, we consider an non-rectangular acoustic pulse, of the form $\sin ^{2}(\omega t)$, lasting $t_{e}=200 \mathrm{~ms}$ with an amplitude $P^{+}=10^{-5}$ (shown in figure $7 a$ ). The reflection coefficients at the inlet and outlet are taken to be $R_{1}=0.75$ and $R_{2}=0.75+0.65 i$ (complex reflection coefficient). The results are shown in figure 10 for $(a)$ OSCILOS, (b) the analytical method using a rectangular approximation with $\delta t=20 \mathrm{~ms}$ and (c) the analytical method using a rectangular approximation with $\delta t=5 \mathrm{~ms}$.

The acoustic pressures computed using OSCILOS and the analytical method show an excellent agreement overall. Although the shape of the acoustic pressure signal obtained using OSCILOS is not entirely replicated for $\delta t=20 \mathrm{~ms}$, this small error becomes negligible for $\delta t=5 \mathrm{~ms}$. The time step $\delta t$ used in the semi-analytical method can be further reduced to achieve results virtually identical to the low-order simulations. Unlike case IV, the acoustic signal contains a negative pressure fluctuation even though the forcing signal is positive. This is because $R_{2}$ is complex valued; the introduction of a complex reflection coefficient introduces oscillations not present in the original acoustic signal.

\section{Test case: Cambridge entropy wave generator}

The Cambridge entropy wave generator experiment, as described in De Domenico et al. (2017), is used as a test case for the analytical results presented in the previous sections. In particular, we are interested in the case where the orifice plate is subsonic, 


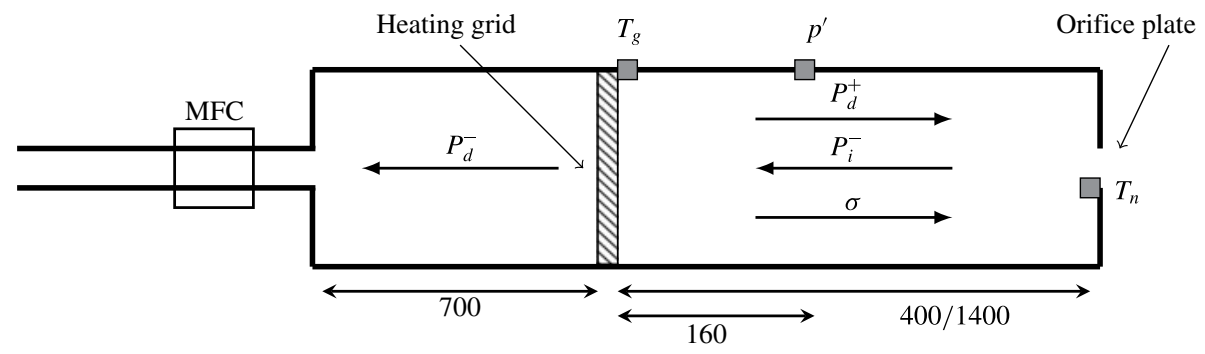

FIGURE 11. Diagram of the Cambridge entropy wave generator with grid and nozzle temperature transducers $\left(T_{g}\right.$ and $\left.T_{n}\right)$ and pressure measurement $p^{\prime}$. All dimensions in $\mathrm{mm}$.

$\begin{array}{lcccccc}\text { Case } & \bar{p}(\mathrm{kPa}) & \bar{u}\left(\mathrm{~m} \mathrm{~s}^{-1}\right) & \bar{M}_{t} & \Delta T_{g}(\mathrm{~K}) & \Delta T_{s}(\mathrm{~K}) & \Delta T_{l}(\mathrm{~K}) \\ 1 & 102.78 & 0.88 & 0.144 & 26.7 & 19.1 & 7.9 \\ 2 & 103.54 & 1.12 & 0.177 & 21.1 & 16.1 & 8.2 \\ 3 & 104.00 & 1.24 & 0.194 & 19.5 & 15.1 & 8.0 \\ 4 & 105.19 & 1.49 & 0.233 & 15.9 & 12.5 & 7.5 \\ 5 & 106.64 & 1.75 & 0.272 & 13.3 & 10.9 & 6.8 \\ 6 & 108.34 & 1.98 & 0.311 & 11.7 & 9.9 & 6.1 \\ 7 & 110.86 & 2.27 & 0.361 & 10.5 & 8.5 & 5.9 \\ 8 & 113.65 & 2.56 & 0.409 & 9.2 & 7.6 & 5.7\end{array}$

TABLE 6. Operating conditions for the short- and long-tube configurations: mean pressure at the grid $\bar{p}$, mean velocity at the grid $\bar{u}$, mean Mach number at the throat $\bar{M}_{t}$, peak hot spot temperature at the grid $\Delta T_{g}$, peak hot spot temperature at the outlet (short tube) $\Delta T_{S}$, peak hot spot temperature at the outlet (long tube) $\Delta T_{L}$.

which corresponds to case $\mathrm{C}$ in the original study. Given that the heat release pulse delivered by the Cambridge EWG is not perfectly rectangular, the method described in $\S 3.5$ is used, whereby the heat release and entropy wave pulses $q^{\prime}$ and $\sigma$ are approximated using a succession of rectangular waves. The duration of these rectangular waves is set to $\delta t=5 \mathrm{~ms}$, which provides an excellent approximation.

\subsection{Experimental set-up}

The Cambridge EWG consists of a $42.6 \mathrm{~mm}$ diameter tube fitted with an electric heater which generates direct noise and entropy waves, as shown in figure 11. The heater is activated for $200 \mathrm{~ms}$ at a time, with a period of $3 \mathrm{~s}$. The tube is terminated with a orifice plate in order to accelerate the flow, generating indirect noise. The diameter of the orifice is $6.6 \mathrm{~mm}$. The system is modular, which allows the convective length to be set to $0.4 \mathrm{~m}$ or $1.4 \mathrm{~m}$.

Temperatures are measured immediately downstream of the heating grid, and at the orifice $\left(T_{g}\right.$ and $T_{n}$ respectively). Pressure fluctuations are measured using a Kulite XTE-190(M) pressure transducer, placed $160 \mathrm{~mm}$ downstream of the heating grid. The mean pressure is measured using a Kulite XT-140M pressure transducer. Measurements are carried out for eight operating conditions, which are presented in table 6.

The experimental set-up is modelled as a tube of constant cross-section with acoustic reflections and the inlet and outlet, direct noise generation at the heating 
grid and indirect noise generation at the outlet. The heating grid consists of three grids of thin tungsten wires $(58 \mu \mathrm{m}$ diameter) placed perpendicular to the flow. Since the change in cross-section due to the wires is less than $0.15 \%$, and the grid is only $10 \mathrm{~mm}$ thick, the grid can be considered acoustically compact and generates no acoustic reflections. This corresponds to the set-up shown in figure 4 with $L_{1}=700 \mathrm{~mm}, x=160 \mathrm{~mm}$ and $L_{2}=400$ or $1400 \mathrm{~mm}$ for the short- or long-tube configuration respectively.

\subsection{Acoustic boundary conditions}

As shown in $\S 4$, the reflection coefficients at the inlet and outlet of the system have a large effect on its acoustic response. As such, acoustic boundary conditions at the inlet and outlet of the tube representative of the real system must be obtained to perform accurate simulations.

The inlet reflection coefficient is defined as $R_{1}=P^{+} / P^{-}$where $P^{+}$is the amplitude of the reflected acoustic wave of an impinging backward-propagating wave $P^{-}$. We assume there are no mass flow rate fluctuations at the mass flow controller $\left(\dot{m}^{\prime}=0\right)$, a condition which is easily met at the current low frequencies. As shown in $\S 2$, this condition can be written as a relationship between acoustic and entropy waves at the mass flow controller location:

$$
\left(1+\frac{1}{\bar{M}}\right) P^{+}+\left(1-\frac{1}{\bar{M}}\right) P^{-}-\sigma=0 .
$$

If we assume that there are no entropy waves upstream of the mass flow controller $(\sigma=0)$, then the reflection coefficient at the mass flow controller can be expressed as:

$$
R_{1}=\frac{P^{+}}{P^{-}}=\frac{1-\bar{M}}{1+\bar{M}},
$$

which corresponds to a reflection coefficient very close to unity for the Mach number range considered here.

Acoustic reflections also occur at the transition between the mass flow controller flexible tube (12 $\mathrm{mm}$ inner diameter) to the main tube $(42.6 \mathrm{~mm}$ inner diameter). Seen from the perspective of an upwards propagating wave $P^{-}$, this change in cross-section can be shown to have a reflection coefficient $R \approx 0.85$. Given that the distance between this transition and the mass flow controller is small relative to the perturbation wavelength (compactness assumption) we assume that both reflections can be captured by the reflection coefficient at the mass flow controller $R_{1}$.

At the outlet of the tube, a free jet is formed due to flow separation at the orifice plate, as shown in figure 12. The jet contracts from an initial cross-section $S_{o}$ to a final cross-section $S_{j}$, where $S_{o}$ and $S_{j}$ are related by the vena contracta factor $\Gamma=S_{j} / S_{o}$. De Domenico et al. (2017) measured the vena contracta factor as 0.83 .

The reflection coefficient of an orifice plate at low frequencies can be predicted with a quasi-steady theory (Durrieu et al. 2001), giving:

$$
R_{2}=-\frac{(1-\bar{M})\left[1-\left(S_{t} / S_{j}\right)^{2}\left(\rho_{t} / \rho_{j}\right)^{2} \bar{M}\right]}{(1+\bar{M})\left[1+\left(S_{t} / S_{j}\right)^{2}\left(\rho_{t} / \rho_{j}\right)^{2} \bar{M}\right]},
$$

where $S_{t}$ and $\rho_{t}$ are the cross-section and the mean density in the tube. If we assume that the jet density $\rho_{j}$ can be taken as atmospheric, then the reflection coefficient at the orifice plate can be readily calculated. The reflection coefficients at the inlet and outlet of the experimental set-up are shown in figure 13 for the all the operating conditions considered here. 


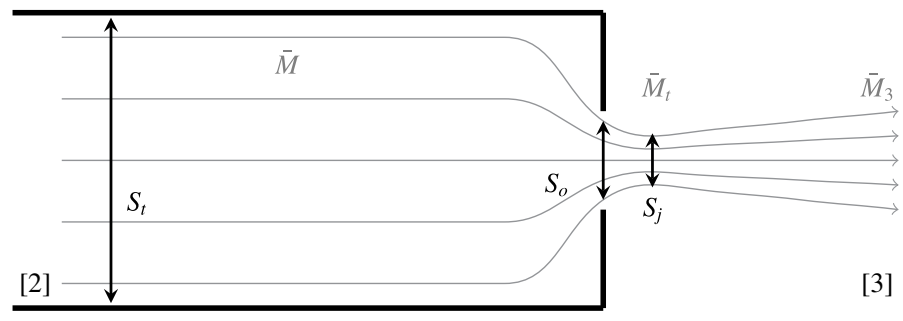

FIGURE 12. Diagram of the flow at the orifice plate with tube, orifice and jet cross-sections $S_{t}, S_{o}$ and $S_{j}$. The flow accelerates from $\bar{M}$ upstream of the orifice [2], forms a jet with maximum Mach $\bar{M}_{t}$ at the vena contracta, and expands to $\bar{M}_{3}$ further downstream [3].

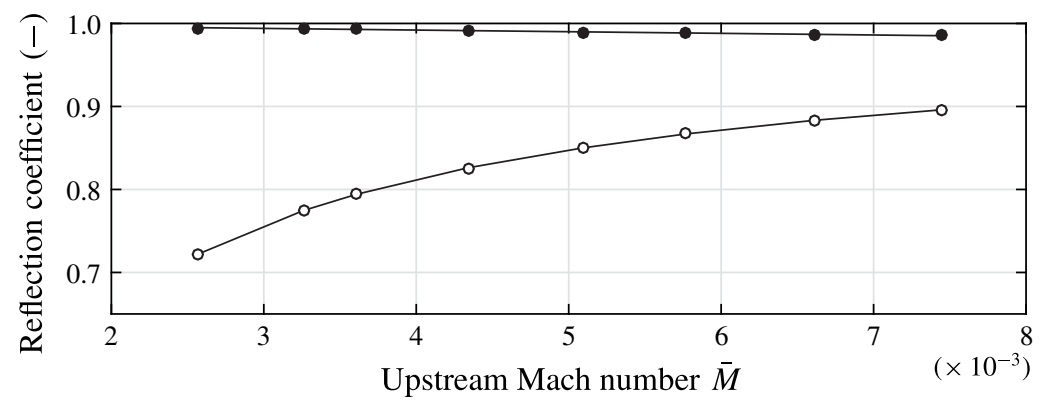

FIGURE 13. Theoretical reflection coefficients at the inlet $R_{1}$ (black circles on solid line, equation (5.2)) and outlet $R_{2}$ (white circles on solid line) of the Cambridge EWG system for cases $1-8$.

\subsection{Acoustic attenuation}

In addition to acoustic losses at the boundaries of the system due to imperfect reflections, there is some acoustic attenuation in the system due to damping effects, transmission through the pipe walls, etc. These are taken into account with a single acoustic attenuation coefficient $\alpha$ (defined in (3.17)).

The amplitude of $\alpha$ can be estimated by examining the experimental results for case B in De Domenico et al. (2017), in which acoustic waves are generated in the absence of air flow, and the system is terminated by rigid walls at both ends. This corresponds to a fully reflective system, where $R_{1}=R_{2}=1$. In the absence of acoustic attenuation, one would expect a gradual acoustic pressure build-up, followed by a constant pressure perturbation (as shown in limit case II). Instead, the experimental results show that there is a slow decay of the acoustic pressure, which corresponds to a loss of acoustic energy, as shown in figure 14 .

The pressure reaches a maximum $p_{m}$ at time $t_{m}$. As shown in (3.18), the decay of the acoustic pressure due to attenuation can be described as $p(t)=p_{m}\left(\mathrm{e}^{-2 \alpha L}\right)^{\left(t-t_{m}\right) / T}$, where $p(t)$ is the value of the decayed pressure at time $t$. In this case, the attenuation coefficient $\alpha$ can be computed as:

$$
\alpha=\frac{\ln \left(p_{m} / p(t)\right)}{\left(t-t_{m}\right) \bar{c}} .
$$

Evaluating $\alpha$ for $t=t_{10 \%}$ (the time at which the pressure has decayed to $10 \%$ of its original value, such that $p_{10 \%} / p_{m}=10 \%$ ) gives $\alpha \approx 0.007 \mathrm{~m}^{-1}$. 


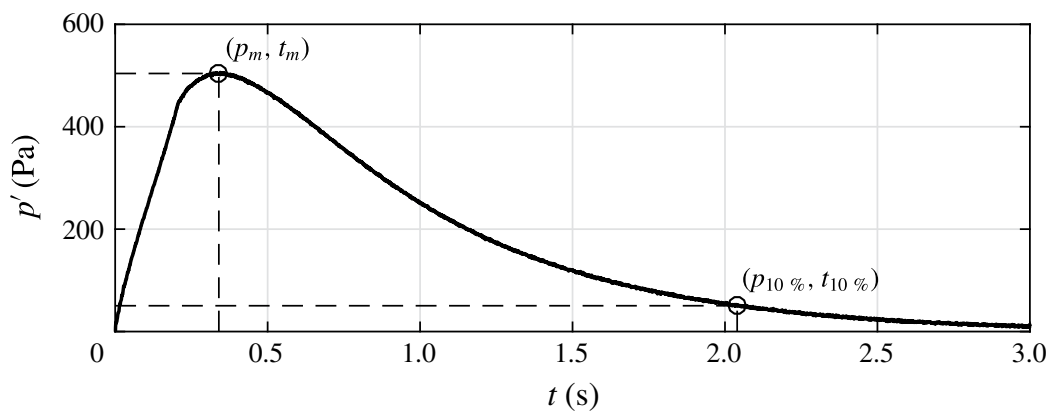

FIgURE 14. Acoustic pressure fluctuation history for case B in De Domenico et al. (2017) (solid line). The coordinates of maximum and decayed pressure $\left(p_{m}, t_{m}\right)$ and $\left(p_{10 \%}, t_{10 \%}\right)$ are circled, and indicated with dashed lines.

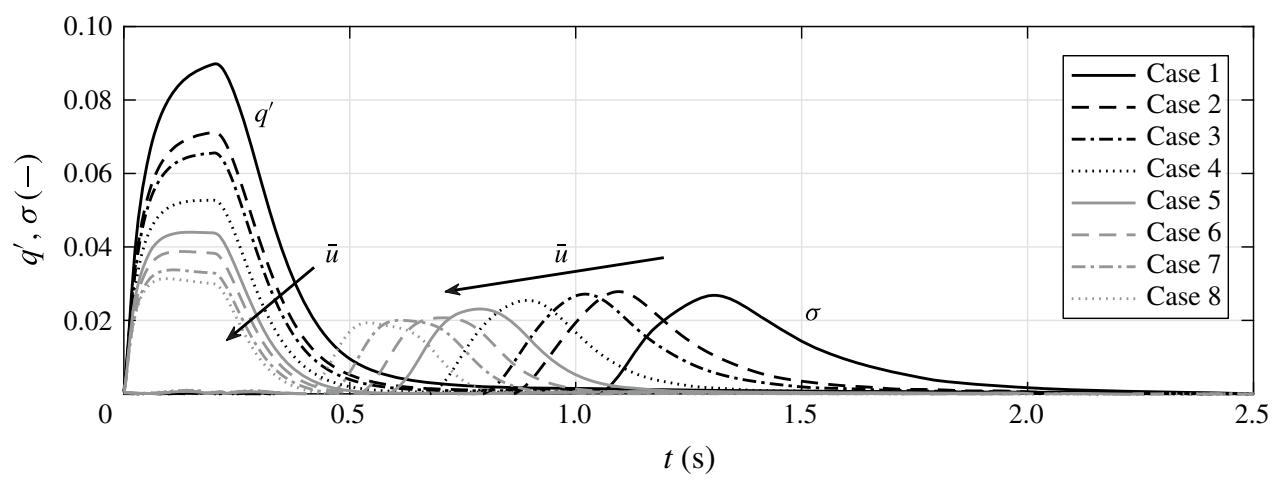

FIGURE 15. Reconstructed normalised fluctuating heat release $q^{\prime}$ and outlet entropy wave amplitude $\sigma$ for cases $1-8$. Arrows indicate the direction of increasing mean velocity $\bar{u}$.

\subsection{Direct and indirect noise models}

Direct and indirect noise are computed using the corresponding transfer functions ( $H_{d}^{+} H_{d}^{-}$and $H_{i}^{-}$respectively). These require knowledge of the heat release fluctuation at the heating grid $q^{\prime}$ and of the entropy wave amplitude at the orifice plate $\sigma$. These inputs are obtained from experimental temperature measurements, as described in De Domenico et al. (2017), and shown for cases 1-8 in figure 15.

As the flow approaches the orifice, it is accelerated and forms a jet downstream. The flow reaches its maximum velocity in the vena contracta, after which point the flow expands and decelerates as it mixes with surrounding air, as shown in figure 12 . This is broadly comparable to the flow through a converging-diverging nozzle.

There are no models in the literature for the entropy noise produced at an orifice plate; the only analytical models available are for isentropic nozzles. For comparison, the model we implement here is the one for an isentropic nozzle of dimensions comparable to that of the orifice plate.

The entropy noise model derived for a 1-D compact isentropic nozzle by Marble \& Candel (1977) was extended by Durán \& Moreau (2013) to non-compact nozzles. In theory, these two models give two different results due to the non-zero frequency of the entropy wave. However, in the case of this experiment, performing a Fourier analysis of the entropy wave signals (shown in figure 15) shows that the frequency 


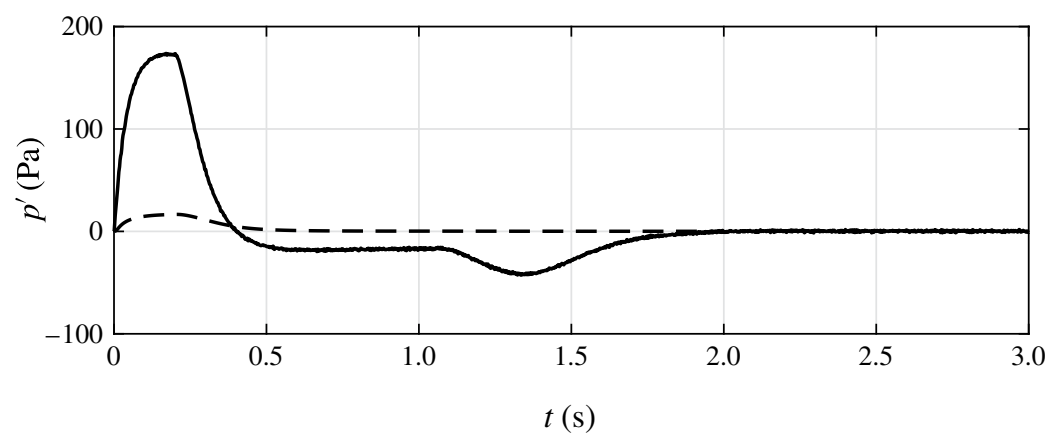

FIGURE 16. Acoustic pressure fluctuation history for case 1 in the subsonic long-tube configuration. Experimental measurement (solid line), analytical result with no acoustic reflections $\left(R_{1}=R_{2}=0\right)$ (dashed line).

content of the entropy waves above $5 \mathrm{~Hz}$ is virtually zero. At such a low frequency, the transfer functions obtained using both models are essentially identical both in phase and amplitude. In our particular case, they differ by less than $0.1 \%$ in amplitude.

The isentropic nozzle model is defined by the Mach numbers upstream and downstream of the nozzle throat, $\bar{M}$ and $\bar{M}_{3}$ respectively. The corresponding indirect noise transfer function $H_{i}^{-}$can be obtained from (2.7).

For the subsonic case, $\bar{M}$ can be taken as the Mach number in the duct, but the downstream Mach number $\bar{M}_{3}$ is not clearly defined for the case of an orifice plate. As such, we consider the range of possible values for $\bar{M}_{3}$, which is bounded by $\bar{M}_{3}=0$ (no flow downstream of the orifice), and by the Mach number in the vena contracta $\bar{M}_{t}$. This enables us to define a range of indirect noise transfer functions $H_{i}^{-}$for $0 \leqslant \bar{M}_{3} \leqslant \bar{M}_{t}$. This range of transfer functions contains all of the values that $H_{i}^{-}$can take for a nozzle comparable to our orifice plate.

For conciseness, the results presented in $\$ 5.5$ are those for the limit case in which $\bar{M}_{3}=\bar{M}_{t}$, which corresponds to a purely converging nozzle. This is the upper limit for the entropy noise which can be produced by a isentropic nozzle of these dimensions. The full range of indirect noise transfer functions is considered in $\S 5.6$.

\subsection{Results}

Figure 16 shows the results for case 1 in the long-tube configuration when acoustic reflections are not taken into account $\left(R_{1}=R_{2}=0\right)$. Neither the shape nor the amplitude of the experimental pressure signal are recovered analytically, and the experimental noise level is an order of magnitude times larger than predicted. While there is a small indirect noise contribution in the analytical signal, it is too small to be seen. The negative pressure fluctuation visible in the experimental results from $t=0.45 \mathrm{~s}$ to $t=1.15 \mathrm{~s}$ is not recovered in the analytical signal. This is consistent with the hypothesis that this negative fluctuation is due to a mean-flow effect, rather than an acoustic one, as suggested by De Domenico et al. (2017).

Figure 17 shows the results for cases 1-8 in the long-tube configuration when acoustic reflections are taken into account.

In their paper, De Domenico et al. (2017) argued that the positive pressure fluctuation could be attributed to direct noise, whereas the time-delayed pressure 

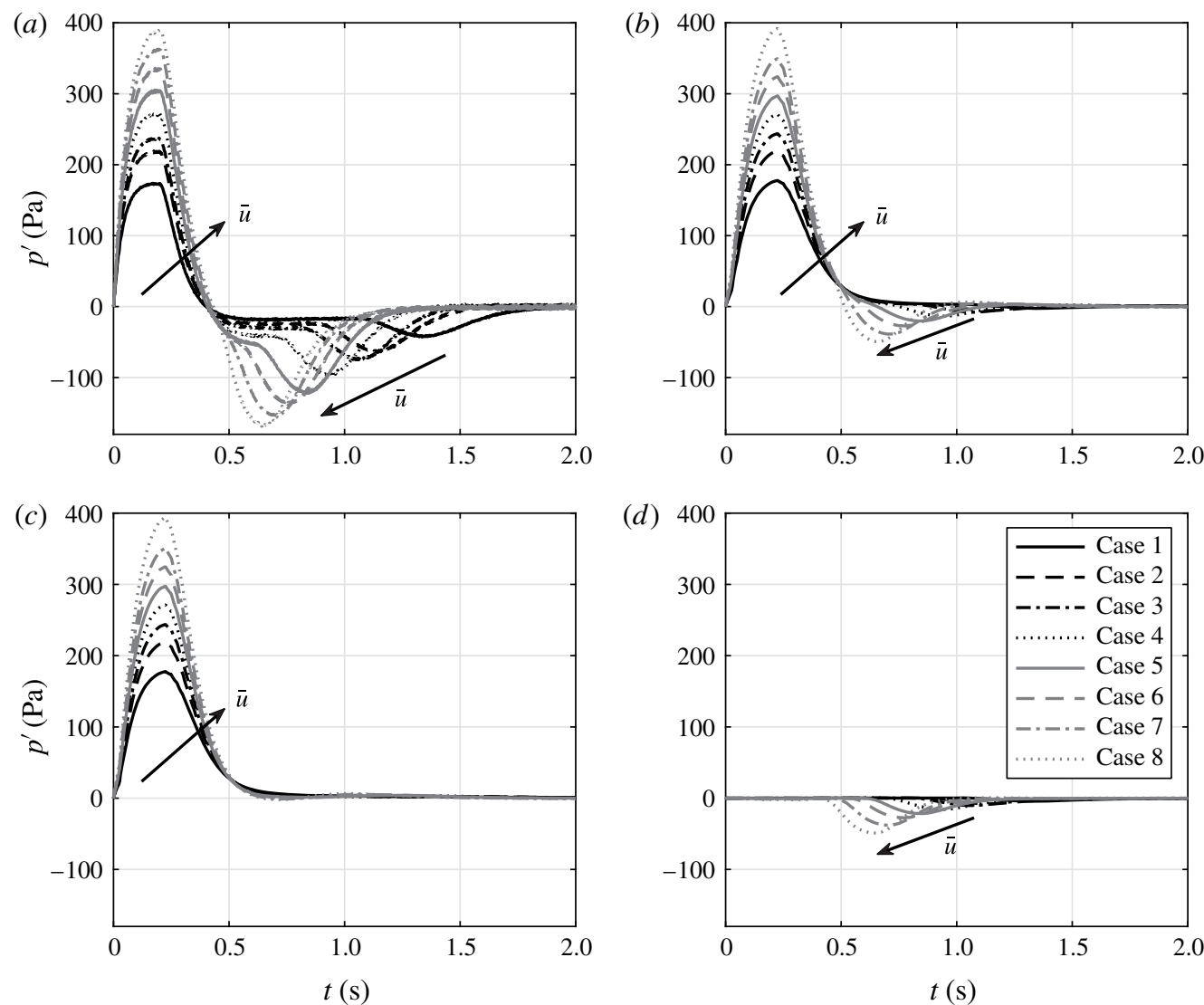

FIgURE 17. Acoustic pressure fluctuation histories for cases 1-8 in the subsonic longtube configuration. (a) Experimental measurement, $(b)$ analytical result, $(c)$ analytical result (direct noise only), $(d)$ analytical result (indirect noise only).

fluctuation could be attributed to indirect noise. The analytical results appear to confirm this; when only direct noise is considered, only the positive pressure fluctuation is recovered, and when only indirect noise is considered, only the time-delayed negative pressure fluctuation is recovered. Once again, the negative pressure fluctuation attributed to a mean-flow effect is not recovered.

The results for direct noise are in good agreement both in shape and amplitude. The increase in direct noise as the upstream Mach number $\bar{M}$ is increased is correctly predicted. This suggests that the analytical model for direct noise and its reverberation are accurate.

Conversely the indirect noise amplitude is significantly under-predicted, although the increase in indirect noise with increasing Mach number is captured in the analytical results. Additionally, the time at which indirect noise is generated is consistent with experimental results, whereby it appears earlier in time for higher Mach numbers as the convective time $\tau_{c}$ decreases. The significant under-prediction of the indirect noise amplitude indicates that the theoretical model for indirect noise generation is not representative of the experiment. The fact that the simulated direct noise is in good agreement with the experimental data indicated that the reverberation model is not at fault. This would mean that the entropy noise model employed here (derived 

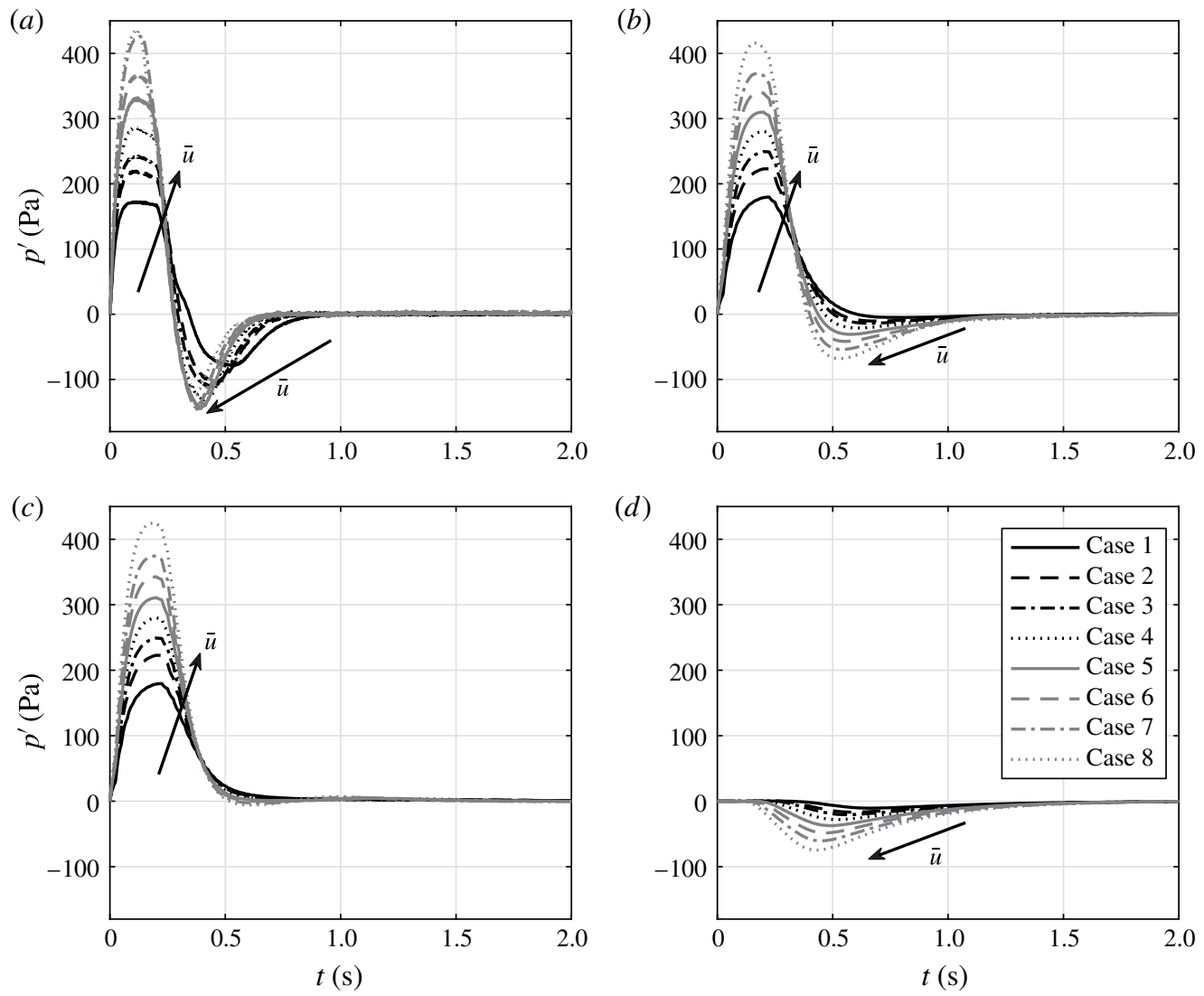

FIGURE 18. Acoustic pressure fluctuation histories for cases 1-8 in the short-tube configuration. (a) Experimental measurement, $(b)$ analytical result, $(c)$ analytical result (direct noise only), $(d)$ analytical result (indirect noise only).

for a 1-D isentropic nozzle) is not adapted to an orifice plate, which is not necessarily isentropic.

Overall, the pressure in the tube appears to rise and decay more sharply than predicted analytically. This may be due to the nature of temperature signal which is used to determine the heat release and entropy wave amplitude at the grid and nozzle respectively. If the shape of this signal is inaccurate (e.g. due to excessive thermal inertia in the thermocouples), then the shape of the simulated pressure signal would be correspondingly affected.

Figure 18 shows the results for cases 1-8 in the short-tube configuration when acoustic reflections are taken into account. Simulations carried out for only direct and indirect noise indicate that the total pressure signal is a combination of direct and indirect noise, with both signals overlapping significantly in the time domain.

The simulated direct noise shows a reasonable agreement with experimental data for all cases. The acoustic pressure levels are slightly higher than for the corresponding cases in the long-tube configuration. This is explained by the fact that in the shorttube configuration, the acoustic time scale $T$ is reduced, resulting in more acoustic reflections in a given time interval, and thus a larger overall pressure fluctuation (as shown in (3.13)). 


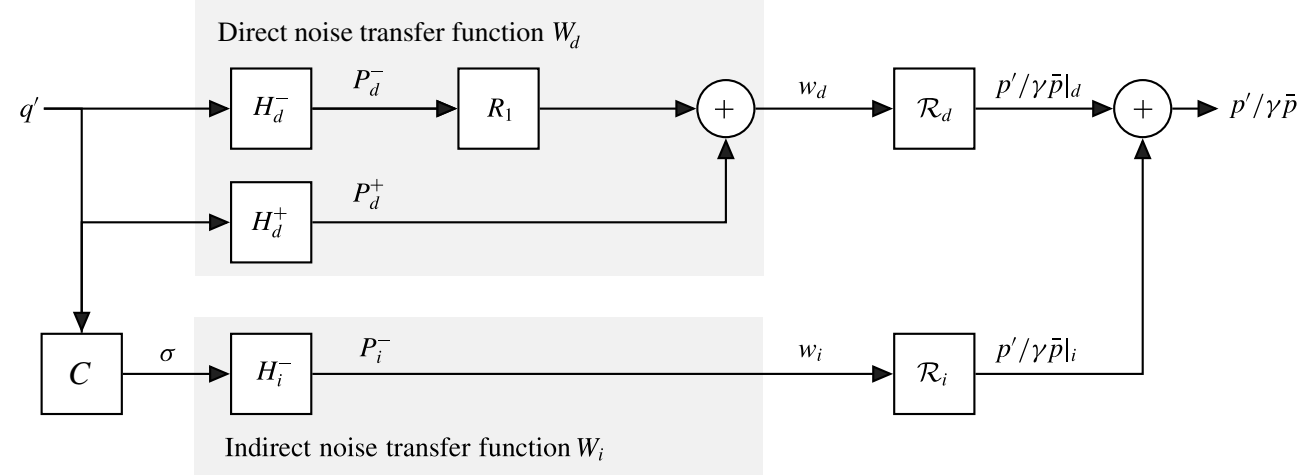

FIGURE 19. Block diagram representation of the relationship between the fluctuating heat release at the grid $q^{\prime}$ and the acoustic pressure fluctuations in the tube $p^{\prime} / \gamma \bar{p} \mid$.

Once again, the indirect noise appears to be significantly under-predicted, but this is hard to determine with certainty, as direct and indirect noise are merged in the experimental results. The negative pressure fluctuation due to the mean-flow effect (visible in the long-tube cases) is likely to be present in the experimental results, but is merged with the indirect noise here. Finally, the indirect noise amplitude is significantly larger than in the long-tube case, in which entropy wave dispersion and dissipation are more prominent due to the increased convective length.

\subsection{Comparison to theoretical models}

The relationship between the heat release $q^{\prime}$ and pressure fluctuations in the tube $p^{\prime} / \gamma \bar{p} \mid$ is shown as a block diagram in figure 19. $C$ is the transfer function between the heat release and the entropy wave at the outlet, taking into account convective time delay, dispersion and dissipation. The effect of reverberation on direct and indirect noise are represented with the transfer functions $\mathcal{R}_{d}$ and $\mathcal{R}_{i}$ respectively.

A meaningful comparison between experimental and theoretical results can only be drawn by comparing quantities independent of factors specific to the experimental set-up, such as acoustic reflections, heat release and convective effects. As such, we define direct and indirect noise transfer functions $W_{d}$ and $W_{i}$, which give a measure of the amount of direct and indirect noise which can be measured downstream of the heating grid. These transfer functions are independent of the effect of both entropy wave dissipation and reverberation:

$$
W_{d}=\frac{w_{d}}{q^{\prime}}=H_{d}^{+}+R_{1} H_{d}^{-}, \quad W_{i}=\frac{w_{i}}{\sigma}=H_{i}^{-} .
$$

Experimental values of these transfer functions can be obtained based on experimental measurements of the entropy and heat release amplitudes $\sigma$ and $q^{\prime}$, as well as the direct and indirect acoustic pressures measurements $\left(p^{\prime} /\left.\gamma \bar{p}\right|_{d, e}\right.$ and $p^{\prime} /\left.\gamma \bar{p}\right|_{i, e}$ respectively) as:

$$
W_{d, e}=\frac{p^{\prime} /\left.\gamma \bar{p}\right|_{d, e}}{q^{\prime} \mathcal{R}_{d}}, \quad W_{i, e}=\frac{p^{\prime} /\left.\gamma \bar{p}\right|_{i, e}}{\sigma \mathcal{R}_{e}} .
$$

The reverberation transfer functions $\mathcal{R}_{d}$ and $\mathcal{R}_{i}$ can be obtained analytically using the method outlined in $\S 3$. The experimental direct and indirect noise transfer 

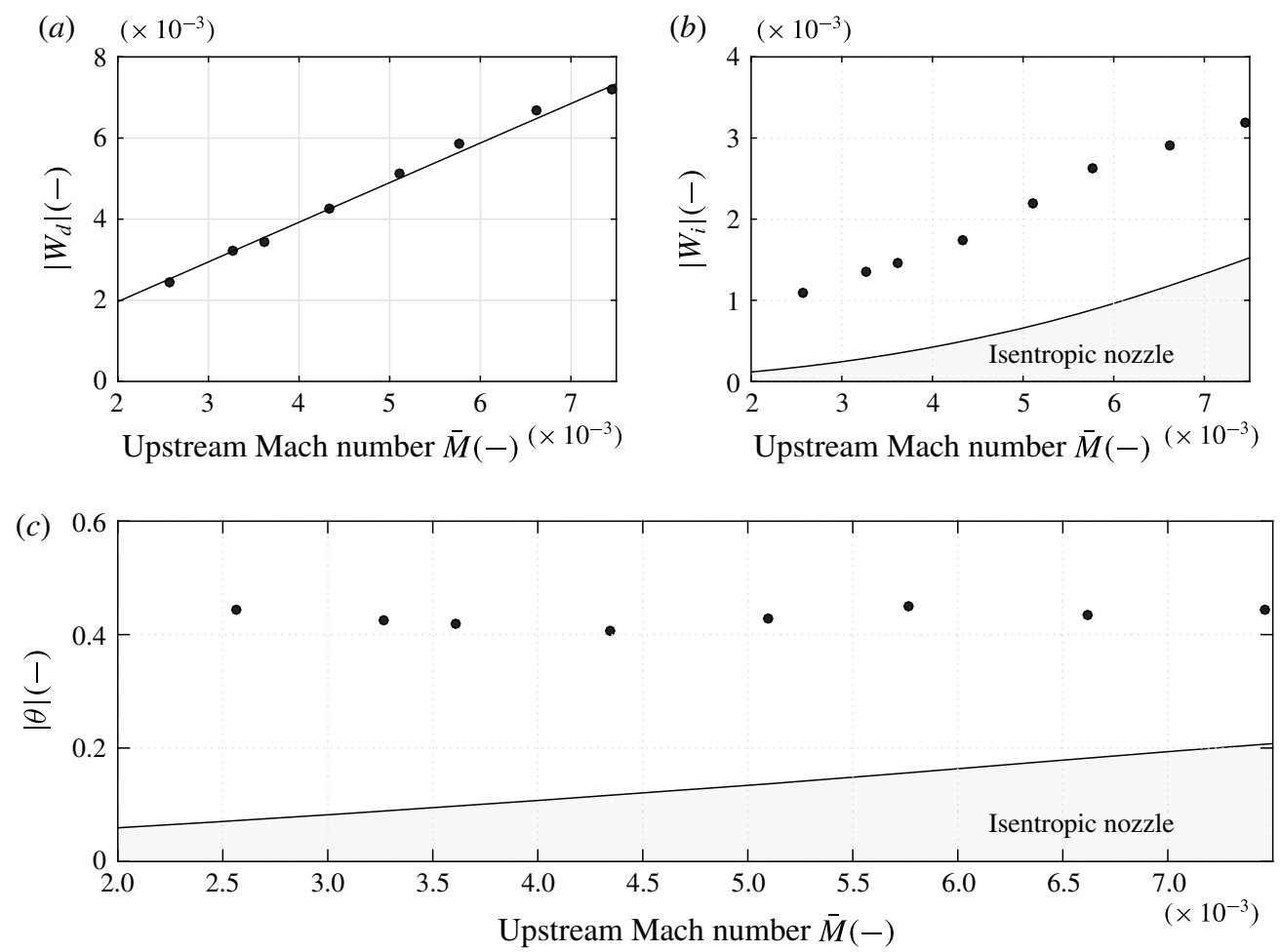

FIgURE 20. (a) Direct noise transfer function $W_{d}$ obtained theoretically (solid line) and experimentally (dots) $(b)$ indirect noise transfer functions $W_{i}$ and $(c)$ ratio of indirect to direct transfer functions $\theta$ obtained theoretically for an isentropic nozzle (shaded area) and experimentally (dots).

functions $W_{d, e}$ and $W_{i, e}$ can be compared to their theoretical values. The theoretical direct noise transfer function $W_{d}$ is based on the model shown in (2.5) and (2.6). The theoretical indirect noise transfer function $W_{i}$ is the one for a 1-D isentropic nozzle, shown in (2.7). $W_{i}$ is computed for a range of Mach numbers downstream of the orifice between $0 \leqslant \bar{M}_{3} \leqslant \bar{M}_{t}$. These limits delimit the full range of possible transfer functions for a 1-D isentropic nozzle of dimensions equivalent to the orifice plate used in the experiments. These are plotted as a function of the upstream Mach number for cases 1-8 in figure 20(a,b).

The experimental and theoretical results for the direct noise transfer function $W_{d}$ are in very good agreement for cases 1-8. This suggests that the theoretical model for direct noise is correctly capturing the physics of the experiment.

The experimentally measured indirect noise transfer function of an orifice plate $W_{i, e}$ is well outside of the range of possible transfer functions for an isentropic nozzle of equivalent dimensions. The discrepancy is most pronounced at low Mach numbers (relative difference of $83 \%$ for case 1), but still significant at higher Mach numbers (relative difference of $50 \%$ for case 8 ).

Experimental values for the indirect to direct noise ratios $\eta=w_{i} / w_{d}$ and $\varphi=p_{i}^{\prime} / p_{d}^{\prime}$ defined in (3.15) and (3.16) can be readily obtained. However, the amplitudes of these transfer functions are affected by entropy wave dispersion and dissipation in the experimental system. An indirect to direct transfer function ratio $\theta$ can be defined, 
which is independent of these effects:

$$
\theta=\frac{W_{i}}{W_{d}}=\frac{q^{\prime}}{\sigma} \eta .
$$

Experimental values for this ratio may be obtained as $\theta_{e x}=W_{i, e} / W_{d, e}$, and compared to the theoretical value for $\theta$ as shown in figure 20(c) for cases 1-8. The amplitude of the experimental indirect to direct transfer function ratio $\theta_{e x}$ is much larger than predicted by the compact nozzle model for all cases. This is a direct consequence of the fact that the amplitude of the indirect noise transfer function $W_{i, e}$ is substantially larger than predicted. Additionally, although we expect the ratio to increase as the upstream Mach number $\bar{M}$ increases, the experimental results do not appear to follow this trend.

\subsection{Discussion}

The experimental results show that in all cases (1-8), the indirect noise produced by an orifice plate is significantly underestimated by the model proposed by Marble \& Candel (1977) and Durán \& Moreau (2013) for a 1-D isentropic nozzle. Possible explanations for this discrepancy may be that (i) there is an additional sound source not accounted for in the analytical model or (ii) an orifice plate generates more entropy noise than an equivalent isentropic nozzle. Both of these explanations are examined here.

First, one may suggest that the negative pressure fluctuation we identify as indirect noise is actually a combination of entropy noise and noise from another source. One candidate for this additional noise source is identified by Howe (2010). He showed that if a jet is formed downstream of the throat (as is the case for a non-isentropic nozzle with flow separation, or for an orifice plate), the vorticity in the jet shear layer is modulated by the passage of an entropy wave, leading to the generation of vortical noise. His study suggests that the vortical noise could manifest itself as an acoustic wave with a negative amplitude, which if transmitted from the jet backwards into the duct could increase the 'perceived' indirect noise. However, the magnitude of this effect is hard to estimate.

Alternatively, the discrepancy between $W_{i, e}$ and $W_{i}$ can be attributed to the differences between an orifice plate and a 1-D isentropic nozzle as considered by Marble \& Candel (1977) and Durán \& Moreau (2013). Intuitively, given that there are substantial losses at an orifice plate, one could expect to obtain less indirect noise than in the isentropic nozzle case. However, this study indicates the opposite may be true. This result is important, as many flow restrictions in real systems are not isentropic. As such the entropy noise produced by these flow elements may be larger than predicted by current theoretical models.

\section{Conclusions and outlook}

Analytical models for the direct noise generated at a heating grid and for the indirect noise generated at an isentropic nozzle are presented. The effect of repeated acoustic reflections (reverberation) on direct and indirect noise generated inside an EWG is then studied analytically. In the simplified case where the frequency of the acoustic source is very low, simple expressions are derived for the evolution of the acoustic pressure with time. These results are implemented in a series of limit cases, showing that acoustic reverberation can significantly attenuate or amplify the original 
signal, and that this effect may affect direct and indirect noise differently. These above results are applied to the Cambridge EWG experiment, in which hot spots are produced with a heating grid (generating direct noise), and accelerated through an orifice plate (generating indirect noise). Analytical simulations of eight cases are performed, employing the indirect noise model derived for a 1-D compact isentropic nozzle by Marble \& Candel (1977) for comparison. Good agreement is found between experimental and simulation results for direct noise. Conversely, the entropy noise generated experimentally at the orifice plate is shown to be significantly larger than predicted by 1-D isentropic nozzle models. For the first time, noise transfer functions can be extracted from the experimental data and compared to theoretical models. The relative error of the isentropic nozzle entropy noise model compared to the experimental results for an orifice plate ranges from $50 \%$ to $83 \%$ depending on the case. This discrepancy is not explained by frequency effects (non-compactness). This suggests that an orifice plate generates more entropy noise than an equivalent isentropic nozzle. Work is currently underway to develop an indirect noise model for orifice plates and non-isentropic nozzles.

\section{Acknowledgements}

This work was supported by the UK Engineering and Physical Sciences Research Council (EPSRC) grant EP/K02924X/1. E.O.R. is supported by an EPSRC DTA studentship (University of Cambridge), and F.D.D. is supported by the Honorary Vice-Chancellor's Award and a Qualcomm/DTA Studentship.

\section{REFERENCES}

Bake, F., Richter, C., Mühlbauer, B., Kings, N., Röhle, I., Thiele, F. \& Noll, B. 2009 The entropy wave generator (EWG): a reference case on entropy noise. J. Sound Vib. 326 (3-5), 574-598.

Becerril, C., Moreau, S., Bauerheim, M., Gicquel, L. \& Poinsot, T. 2016 Numerical investigation of combustion noise: the entropy wave generator. In 22nd AIAA/CEAS Aeroacoustics Conference. American Institute of Aeronautics and Astronautics.

BoHn, M. S. 1976 Noise produced by the interaction of acoustic waves and entropy waves with high-speed nozzle flows. PhD thesis, California Institute of Technology.

BragG, S. L. 1963 Combustion noise. J. Inst. Fuel 36 (264), 12-16.

Chu, B. T. \& Kovásznay, L. S. G. 1958 Non-linear interactions in a viscous heat-conducting compressible gas. J. Fluid Mech. 3 (05), 494-514.

Cumpsty, N. A. 1979 Jet engine combustion noise: pressure, entropy and vorticity perturbations produced by unsteady combustion or heat addition. J. Sound Vib. 66 (4), 527-544.

De Domenico, F., Rolland, E. O. \& Hochgreb, S. 2017 Detection of direct and indirect noise generated by synthetic hot spots in a duct. J. Sound Vib. 394, 220-236.

Dowling, A. P. \& Mahmoudi, Y. 2014 Combustion noise. Proc. Combust. Inst. 35 (1), 65-100.

Dowling, A. P.\& Stow, S. R. 2003 Acoustic analysis of gas turbine combustors. J. Propul. Power 19 (5), 751-764.

Durán, I. \& Moreau, S. 2013 Solution of the quasi-one-dimensional linearized Euler equations using flow invariants and the Magnus expansion. J. Fluid Mech. 723, 190-231.

Durán, I., Moreau, S. \& Poinsot, T. 2013 Analytical and numerical study of combustion noise through a subsonic nozzle. AIAA J. 51 (1), 42-52.

Durrieu, P., Hofmans, G., Ajello, G., Boot, R., Auregan, Y., Hirschberg, A. \& Peters, M. C. A. M. 2001 Quasisteady aero-acoustic response of orifices. J. Acoust. Soc. Am. 110 (4), 1859-1872. 
Ffowcs Williams, J. E. \& Howe, M. S. 1975 The generation of sound by density inhomogeneities in low Mach number nozzle flows. J. Fluid Mech. 70 (03), 605-622.

Giauque, A., Huet, M. \& Cléro, F. 2012 Analytical analysis of indirect combustion noise in subcritical nozzles. Trans. ASME J. Engng Gas Turbines Power 134 (11), 1745-1755.

Goh, C. S. \& Morgans, A. S. 2011 Phase prediction of the response of choked nozzles to entropy and acoustic disturbances. J. Sound Vib. 330 (21), 5184-5198.

GoH, C. S. \& Morgans, A. S. 2013 The influence of entropy waves on the thermoacoustic stability of a model combustor. Combust. Sci. Technol. 185 (2), 249-268.

HAKE, M. I. 2014 Experimental design to determine the effect of temperature and Mach number on entropy noise. Master's thesis, Massuachusets Institute of Technology.

Howe, M. S. 2010 Indirect combustion noise. J. Fluid Mech. 659, 267-288.

HUET, M. 2016 Nonlinear indirect combustion noise for compact supercritical nozzle flows. J. Sound Vib. 374, 211-227.

Huet, M. \& Giauque, A. 2013 A nonlinear model for indirect combustion noise through a compact nozzle. J. Fluid Mech. 733, 268-301.

Leyko, M., Moreau, S., Nicoud, F. \& Poinsot, T. 2011 Numerical and analytical modelling of entropy noise in a supersonic nozzle with a shock. J. Sound Vib. 330 (16), 3944-3958.

Leyko, M., Nicoud, F. \& Poinsot, T. 2009 Comparison of direct and indirect combustion noise mechanisms in a model combustor. AIAA J. 47 (11), 2709-2716.

Lourier, J. M., Huber, A., Noll, B. \& Aigner, M. 2014 Numerical analysis of indirect combustion noise generation within a subsonic nozzle. AIAA J. 52 (10), 2114-2126.

Magri, L., O'Brien, J. \& Ihme, M. 2016 Compositional inhomogeneities as a source of indirect combustion noise. J. Fluid Mech. 799, R4.

Marble, F. E. \& CANDEL, S. M. 1977 Acoustic disturbance from gas non-uniformities convected through a nozzle. J. Sound Vib. 55 (2), 225-243.

Moase, W. H., Brear, M. J. \& Manzie, C. 2007 The forced response of choked nozzles and supersonic diffusers. J. Fluid Mech. 585, 281-304.

Morfey, C. L. 1973 Amplification of aerodynamic noise by convected flow inhomogeneities. J. Sound Vib. 31 (4), 391-397.

Mühlbauer, B., Noll, B. \& Aigner, M. 2009 Numerical investigation of the fundamental mechanism for entropy noise generation in aero-engines. Acta Acust. United Acustica 95 (3), 470-478.

Polifke, W., Paschereit, C. O. \& Klaus, D. 2001 Constructive and destructive interference of acoustic and entropy waves in a premixed combustor with a choked exit. Intl J. Acoust. Vib. 6 (3), 135-146.

Stow, S. R., Dowling, A. P. \& Hynes, T. P. 2002 Reflection of circumferential modes in a choked nozzle. J. Fluid Mech. 467, 215-239.

Strahle, W. C. 1971 On combustion generated noise. J. Fluid Mech. 49 (02), 399-414.

Tao, W., Mazur, M., Huet, M. \& Richecoeur, F. 2016 Indirect combustion noise contributions in a gas turbine model combustor with a choked nozzle. Combust. Sci. Technol. 188 (4-5), 793-804.

Zukoski, E. E. \& Auerbach, J. M. 1976 Experiments concerning the response of supersonic nozzles to fluctuating inlet conditions. J. Engng Power 98 (1), 60-64. 